Aus der Klinik für Neurochirurgie

(Prof. Dr. med. V. Rohde)

im Zentrum Neurologische Medizin

der Medizinischen Fakultät der Universität Göttingen

\title{
Die Trigeminusneuralgie als Differentialdiagnose des odontogenen Schmerzes
}

\author{
INAUGURAL-DISSERTATION
}

zur Erlangung des Doktorgrades

für Zahnheilkunde

der Medizinischen Fakultät der

Georg-August-Universität zu Göttingen

vorgelegt von

Moritz Keil

aus

Dortmund

Göttingen 2014 
Dekan: Prof. Dr. rer. nat. H.K. Kroemer

I. Berichterstatter: Prof. Dr. Veit Rohde

II. Berichterstatter: Prof. Dr. Dr. Henning Schliephake

III. Berichterstatter: Prof. Frank Petzke

Tag der mündlichen Prüfung: 07.05.2014 


\section{Inhaltsverzeichnis}

1 Einleitung $\quad 1$

1.1 Stand der Wissenschaft . . . . . . . . . . . . . . . . . . . 2

1.1.1 Geschichte der Erforschung der Trigeminusneuralgie . . . . . . . 2

1.1.2 Klassifikation . . . . . . . . . . . . . . . . . . 4

Klassische Trigeminusneuralgie . . . . . . . . . . . . 4

Symptomatische Trigeminusneuralgie . . . . . . . . . 5

Alternative Einteilung . . . . . . . . . . . . . . . . . 6

Bildgebende Verfahren . . . . . . . . . . . . . 6

1.1.3 Therapieformen . . . . . . . . . . . . . . . . 7

Medikamentöse Therapie . . . . . . . . . . . . . . . . 7

Chirurgische Therapie . . . . . . . . . . . . . . . . 8

1. Posteriore Fossa . . . . . . . . . . . . . . . . . . . . . . 9

2. Ganglion Gasseri-Bereich . . . . . . . . . . . . . 10

3. Periphere Techniken . . . . . . . . . . . . . . . 11

1.2 Fragestellung . . . . . . . . . . . . . . . . . . . . 12

2 Material und Methoden 13

2.1 Anschreiben . . . . . . . . . . . . . . . . . . . . 13

2.2 Trigeminusneuralgiepatienten f . . . . . . . . . . . . . . . 13

2.3 Zahnärzte . . . . . . . . . . . . . . . . . . . . . . . 14

2.4 Fragebögen . . . . . . . . . . . . . . . . . . . . . . . 14

2.4.1 Fragebogen Patienten . . . . . . . . . . . . . . 15

2.4.2 Fragebogen Zahnärzte . . . . . . . . . . . . . . . 16

3 Ergebnisse 18

3.1 Trigeminusneuralgiepatienten f . . . . . . . . . . . . . . . . . . . . 18

3.1 .1 Gesamtgruppe . . . . . . . . . . . . . 18

Initialer Arztkontakt . . . . . . . . . . . . . . . . . 18

Zahnärztliche Evaluation und Maßnahmen . . . . . . . . . . 18

3.1 .2 Untergruppenanalyse . . . . . . . . . . . . . . . . . . . 22

OP-Gruppe I . . . . . . . . . . . . . . . . . . . . 22

OP-Gruppe II . . . . . . . . . . . . . . . . . . . . . . 23 
3.2 Zahnärzte . . . . . . . . . . . . . . . . 25

4 Diskussion $\quad 28$

4.1 Limitationen . . . . . . . . . . . . . . . . . . . . . . . . . . 28

4.2 Diskussion der Ergebnisse . . . . . . . . . . . . . . . . . . . . . . . 28

4.2.1 TN-Patienten gehen als erstes zum Zahnarzt . . . . . . . . . . . 29

4.2.2 TN-Patienten werden zahnmedizinisch behandelt . . . . . . 30

4.2.3 TN-Patienten kommen spät in neurologogische oder neurochirurgische Einrichtungen . . . . . . . . . . . . . . . . 32

4.2 .4 Zahnärzte . . . . . . . . . . . . . . . . . 32

4.2 .5 Schlussfolgerung . . . . . . . . . . . . . . . . . . 33

5 Zusammenfassung $\quad 35$

6 Anhang 37

6.1 Abkürzungsverzeichnis . . . . . . . . . . . . . . . . . . . . 37

6.2 Tabellenverzeichnis . . . . . . . . . . . . . . . . . . . . . . . . . . . 38

6.3 Abbildungsverzeichnis . . . . . . . . . . . . . . . . . 38

6.4 Anschreiben Patienten . . . . . . . . . . . . . . . . . . . . 39

6.5 Fragebogen Patienten . . . . . . . . . . . . . . . . . 41

6.6 Anschreiben Zahnärzte . . . . . . . . . . . . . . . . . . 43

7 Literaturverzeichnis $\quad 45$ 


\section{Einleitung}

Die Trigeminusneuralgie (im Folgenden TN) zählt zu den Krankheiten des Nervensystems und wird als blitzartig einschießender, extrem heftiger, elektrisierender und stechender Schmerz im Versorgungsgebiet eines oder mehrerer Äste des Nervus trigeminus definiert. Der Trigeminusnerv ist der Fünfte (V) der zwölf sogenannten Hirnnerven. Er ist paarig angelegt und teilt sich nach dem Austritt aus der Pons in seine drei Unteräste. Diese sind der Augenast (Nervus ophtalmicus, $V_{1}$ ), der Oberkieferast (Nervus maxillaris, $\mathrm{V}_{2}$ ) und der Unterkieferast (Nervus mandibularis, $\mathrm{V}_{3}$ ), welche für die somatosensible Versorgung der jeweiligen Gesichtshälfte einschließlich der Zähne und die motorische Innervation der Kaumuskulatur verantwortlich sind.

Im Ausbreitungsgebiet dieses Nervs können bei einer TN von sich aus nicht schmerzhafte Auslöser, sogenannte Trigger, wie ein leichter Windzug, Rasieren, Sprechen oder Schlucken, aber auch Kauen, Zähneputzen oder Berührungen in der perioralen Region starke Schmerzen auslösen. Am häufigsten betroffen sind dabei die Äste $V_{2}$ und $V_{3}$.

Die TN ist eine seltene Krankheit mit einem geringen allgemeinen Bekanntheitsgrad. Dem Betroffenen wird dadurch erschwert zu erkennen, was die Ursache seiner Beschwerden ist und was folglich die richtige Einrichtung zur Bekämpfung derselbigen wäre. Der Patient stellt vereinfacht gesagt nur fest, dass er starke Schmerzen im Bereich des Ober- oder Unterkiefers hat, welche durch eine gewöhnliche Schmerzmedikation nicht kontrollierbar sind.

Damit stellt sich die Frage, ob Patienten die genannten Symptome als ein zahnärztliches Problem wahrnehmen und folglich eine zahnmedizinische Einrichtung aufsuchen.

Sollte dies der Fall sein, ist ebenfalls von Interesse, wie ein Zahnarzt mit einer solchen seltenen Nervenkrankheit umgeht.

Der Gesichtsbereich ist sowohl anatomisch als auch neurologisch ein sehr komplexes Gebiet. Selbst für medizinisches Fachpersonal ist die Differentialdiagnostik von unklaren Gesichtsschmerzen eine große Herausforderung.

Stellt sich nun ein Patient mit unklaren Gesichtsschmerzen beim Zahnarzt vor, muss dieser anhand von Anamnese, Befund und weiterführender Diagnostik wie bildgebenden Verfahren eine Verdachtsdiagnose stellen und dementsprechend seine Behandlung planen. Dabei kommen neben odontogenen Auslösern wie Entzündungen und Infektionen der Zahnpulpa oder schmerzhaften Myoarthropathien auch nicht odontogene Ursa- 
chen in Frage. In Betracht gezogen werden müssen vor allem die atypische Odontalgie, Clusterkopfschmerzen, die postzosterische Neuralgie oder aber die Trigeminusneuralgie. Letztendlich steht der Zahnarzt vor der Entscheidung zwischen einer eigenen - möglicherweise invasiven - Behandlung oder einer Überweisung zu einem Behandler, der eine andere Fachdisziplin repräsentiert bzw. andere Behandlungsschwerpunkte setzt. Voraussetzung zum Erkennen einer TN ist das ausreichende Wissen über ihre wesentlichen Merkmale und die diagnostisch wichtigen Kriterien zur differentialdiagnostischen Abgrenzung.

\subsection{Stand der Wissenschaft}

\subsubsection{Geschichte der Erforschung der Trigeminusneuralgie}

Der erste Hinweis auf eine TN wird Aretaios von Kappadokien zugeschrieben. Bekanntgeworden durch eine der frühesten Beschreibungen der Migräne, schrieb er um 200 n. Chr. von einem Kopfschmerz ,in Zusammenhang mit Krämpfen und Verzerrungen des Gesichtes" (Rose 1999).

Im 11. Jahrhundert erwähnte der arabische Arzt Jujani in seinen Schriften einen einseitigen Gesichtsschmerz, der Krämpfe und Angst auslöst (Ameli 1965, Nurmikko und Eldridge 2001).

Die Bezeichnung tic douloureux, welche heute noch teilweise gebräuchlich ist, entstammt der Beschreibung durch Nicolas André aus dem Jahre 1756. Er vertrat die Meinung, dass „bösartige Nervenflüssigkeiten“ den Nerv irritieren und schmerzhafte Stöße verursachen (Cole et al. 2005, Harris 1951). Später stellte sich heraus, dass von den fünf von André vorgestellten Patienten nur zwei tatsächlich eine TN hatten (Rose 1999).

1773 präsentierte der englische Arzt John Fothergrill der Medical Society of London seine Beobachtungen von 14 Patienten. Unter dem Titel „Of a painful affection of the face" gelingt ihm die bis dato beste und ausführlichste Beschreibung der TN. Fothergrill stellte darin eine Krankheit vor, die hauptsächlich bei älteren Frauen in Erscheinung tritt und durch plötzlich einschießende, vernichtend starke Schmerzen charakterisiert wird. Ihm fiel sowohl ein intervallartiges Auftreten bei einer Dauer von durchschnittlich 25 Sekunden auf sowie die Auslösbarkeit durch geringste Muskelbewegungen wie Essen und Sprechen oder einfache Berührungen mit der Hand. Als Ursache vermutete Fothergrill eine Art Tumor (Cole et al. 2005, Prasad und Galetta 2009). Auf Grund seiner guten klinischen Beschreibung wurde die TN von vielen auch als „Fothergrill's disease" bezeichnet.

Für das Verständnis der Erkrankung maßgeblich war auch die Unterscheidung der 
Funktionen des N. trigeminus und des N. facialis durch Charles Bell im Jahre 1829. Diese ließ ihn zu dem Schluss kommen, dass der paroxysmale Schmerz der TN direkt mit einer Funktionsstörung des N. trigeminus in Verbindung steht (Prasad und Galetta 2009, Wilkins und Brody 1969).

Diese Zuordnung führte zu der Entwicklung verschiedenster Operationsmethoden bei denen der N. trigeminus teilweise oder ganz durchtrennt, das Ganglion Gasseri teilweise oder ganz entfernt (Harris 1951) oder ein nerv-stretching durchgeführt wurde (Keane 1990).

Es war Edward Walter Dandy, der in seinem Review „The treatment of trigeminal neuralgia by the cerebellar route“ 1932 anmerkte, dass in vielen Fällen von TN arterielle oder venöse Schleifen im Bereich des Hirnstamms den Nerv lädieren und für die Neuralgie verantwortlich sein könnten (Dandy 1932, Prasad und Galetta 2009). Ähnliche Beobachtungen konnten auch von W. James Gardner um 1960 gemacht werden (Gardner und Miklos 1959, Gardner 1962).

Auf diesen Erkenntnissen aufbauend entwickelte Jannetta (1967) eine vielzitierte Theorie der vaskulären Kompression, die nahelegt, dass es durch den unphysiologischen Gefäß-Nerven-Kontakt (siehe Abbildung 1.1) im Bereich des Kleinhirnbrückenwinkels zu einer Demyelinisierung des N. trigeminus kommt und folglich die Bildung ektopischer Nervenimpulse begünstigt wird (Stienen et al. 2010).

Er postulierte davon abgeleitet eine Operationsmethode, die noch heute nach ihm benannt wird. Die mikrovaskuläre Dekompression nach Jannetta beruht auf dem operativen Abpräparieren des den Trigeminusnerv komprimierenden Blutgefäßes und dem Separieren beider durch ein geeignetes Material, wie zum Beispiel ein Schwämmchen aus Teflon (Jannetta 1980).

Abbildung 1.1: Intraoperative Darstellung eines Gefäß-Nervenkontaktes am N. trigeminus

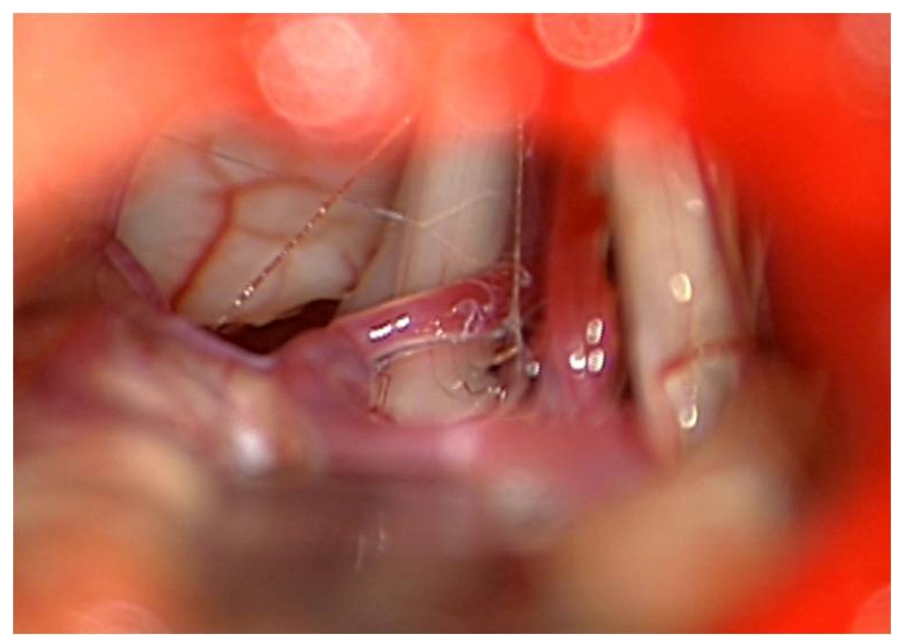




\subsubsection{Klassifikation}

Die TN wird seitens der Weltgesundheitsorganisation (WHO) in der „Internationalen Statistischen Klassifikation der Krankheiten und verwandter Gesundheitsprobleme" in der Verschlüsselung ICD-10-GM von 2006 (Krause 2007) unter der Rubrik „Krankheiten von Nerven, Nervenwurzeln und Nervenplexus" mit dem Kode G 50.0 geführt. Betrachtet man die Klassifikation der International Headache Society (IHS) in ihrer „Internationalen Klassifikation von Kopfschmerzerkrankungen“ in der zweiten Auflage (ICHD-2, Olesen 2006) sowie gängige Lehrbücher und Publikationen, so wird deutlich, dass in der Literatur einerseits zwischen der klassischen (früher: idiopathischen) und andererseits der symptomatischen TN unterschieden wird. Zu erkennen beispielsweise bei der IHS, wo die TN in die Rubrik „Teil 3: Kraniale Neuralgien und zentrale Ursachen von Gesichtsschmerzen" unter 13.1 geführt und unterteilt wird in die klassische Trigeminusneuralgie (13.1.1) und die symptomatische Trigeminusneuralgie (13.1.2).

\section{Klassische Trigeminusneuralgie}

Laut Definition handelt es sich bei der klassischen TN um einen blitzartig einschießenden, extrem heftigen, elektrisierenden und stechenden Schmerz im Versorgungsgebiet eines oder mehrerer Äste des Nervus trigeminus (Gronseth et al. 2008, IASP 1986, Stienen et al. 2010, Zakrzewska 2002). Hierbei ist der Nervus maxillaris am häufigsten, der Nervus ophtalmicus am seltensten betroffen (Katusic et al. 1990, Krafft 2008). Die Attacken halten typischerweise Sekunden, selten auch länger, jedoch per definitionem unter zwei Minuten an (Love und Coakham 2001). Sie können sowohl spontan als auch durch Reize wie Kauen, Sprechen, Schlucken, Rasieren, Zähneputzen oder Berührungen im Versorgungsgebiet des ipsilateralen N. trigeminus ausgelöst werden. Zwischen den Attacken besteht Beschwerdefreiheit die Tage, Monate bis Jahre andauern kann (Zakrzewska 2010 a). Die Neuralgie ist in fast allen Fällen unilateral und der Befund einer neurologischen Untersuchung negativ (Krafft 2008).

Katusic et al. bestimmten für eine 1990 veröffentlichte und häufig zitierte epidemiologische Studie die Inzidenz der TN in Rochester, Minnesota (USA) von 1945 bis 1984. Es ergibt sich eine Inzidenz von 4,3 Neuerkrankungen auf 100.000 Einwohner pro Jahr, wobei Frauen $(5,9)$ häufiger betroffen sind als Männer $(3,4)$. Damit ist die TN den seltenen Krankheiten zuzuordnen. Neuere Untersuchungen von Koopman et al. (2009) beziffern die Inzidenz mittlerweile auf 12,6 pro 100.000 Einwohner mit einer ähnlichen Geschlechterverteilung.

Beide Studien zeigen, dass die Inzidenz mit dem Alter steigt und laut Katusic et al. (1990) einen Häufigkeitsgipfel zwischen dem 50. und 60. Lebensjahr aufweist. Vor dem 40. Lebensjahr tritt die Erkrankung selten auf. Die Prävalenz liegt bei 40 Erkrankten 
pro 100.000 Einwohner. Als Risikofaktor konnte bisher nur die Hypertension identifiziert werden (Zakrzewska 2010 a).

Die Ätiologie der TN konnte bis heute nicht abschließend geklärt werden. So gibt es weiterhin keinen objektivierbaren Test und die Diagnose wird fast ausschließlich anhand von anamnestischen sowie klinischen Kriterien gestellt. Trotzdem gilt als allgemein anerkannt, dass in den meisten Fällen eine vaskuläre Kompression der Nervenwurzel beim Eintritt in die Pons als pathologisches Korrelat dient (Nurmikko und Eldridge 2001).

Differentialdiagnostisch abzugrenzen sind die atypische Odontalgie, der neuropathische Trigeminusschmerz (z.B. Deafferenzierungsschmerz) und primäre odontogene Schmerzsyndrome wie die Pulpitis (Türp 2005, Zakrzewska 2010 a). Ebenfalls in Frage kommen die Sinusitis, Migräne oder andere primäre Kopfschmerzen sowie Myoarthropathien (Baad-Hansen 2008). Je nach Lokalisation der Symptomatik müssen auch postzosterische Neuralgien, Orbitaerkrankungen, die Arteriitis cranialis oder intrakranielle Tumore in Betracht gezogen werden (Zakrzewska 2010 a).

Selten, aber auf Grund einer drohenden Erblindung wichtig, sind die von Cohen et al. (2006) beschriebenen Erkrankungsbilder SUNCT (short unilateral neuralgiform pain with conjunctival redness and tearing) und SUNA (short unilateral neuralgiform pain with autonomic symptoms).

\section{Symptomatische Trigeminusneuralgie}

Die symptomatische TN unterscheidet sich in der Form des Schmerzes nicht von einer klassischen TN. Allerdings konnte festgestellt werden, dass die Patienten zwischen den Attacken teilweise nicht schmerzfrei sind, ein hintergründiges Brennen spüren und sensible Defizite im betroffenen Trigeminusast aufweisen können (Cruccu et al. 2008, Zakrzewska 2010 a). Außerdem können ein bilaterales Vorkommen sowie abnormale Trigeminusreflexe ein Unterscheidungsmerkmal sein (Gronseth et al. 2008).

Der pathologische Unterschied ist eine andere nachweisbare strukturelle Läsion als eine Gefäßkompression, welche pathoanatomisch fassbar ist. Dies können raumfordernde Prozesse wie ein Akustikusneurinom, ein Meningeom oder Metastasen sein. In Frage kommen weiterhin Demyelinisierungen als Folge einer Multiplen Sklerose (Chemaly et al. 2000, Nurmikko 2009, Sarlani et al. 2005) oder pathologische Prozesse im Bereich des Hirnstamms, wie zum Beispiel ein Infarkt (Prasad und Galetta 2009).

Zu erwähnen bleibt, dass die Raumforderungen bei einem Teil der Patienten indirekt ebenfalls zu einem pathologischen Gefäß-Nervenkontakt mit vaskulärer Kompression führen können (Stienen et al. 2010). 


\section{Alternative Einteilung}

Einigen Autoren ist die Unterteilung in klassische und symptomatische TN nicht präzise genug, was zu der Entwicklung alternativer Einteilungen geführt hat. So haben beispielsweise Nurmikko und Eldridge (2001) mit ihren sogenannten „Liverpool criteria" eine Einteilung anhand des Schmerzes in drei Kategorien vorgeschlagen. Sie unterscheiden die typische TN von der atypischen TN und der trigeminalen Neuropathie. Als Kriterien dienen vor allem die Schlüsselelemente Schmerzqualität, Schmerzdauer, betroffene Seite, Allodynie, Auslöser, Sensibilitätsverlust und Schmerzentwicklung.

Eller et al. (2005) propagieren eine differenziertere Einteilung und unterteilen das Krankheitsbild der TN nach sieben diagnostischen Kriterien. Die klassische TN fällt mit ihrer Symptomatik des blitzartig einschießenden, heftigen und stechenden Schmerzes, der episodisch auftritt und wenige Sekunden dauert, in die Diagnosegruppe TN1. Die Diagnosegruppe TN2 umschreibt als diagnostisches Kriterium einen eher brennenden, pochenden Schmerz, der sich vor allem durch seine Konstanz als Hintergrundschmerz geriert und sich aus einer TN1 entwickeln kann. Die Diagnose Nr. 3 beschreibt hingegen den neuropathischen Trigeminusschmerz, verursacht durch eine traumatische oder unbeabsichtigte operative Verletzung des Trigeminusnervs. Ein trigeminaler Deafferenzierungsschmerz, hervorgerufen durch operative Verfahren zur Behandlung von TN oder andere verbundene Gesichtsschmerzen, soll laut den Autoren der Diagnose Nr. 4 zugeordnet werden. Die Diagnose Nr. 5 beinhaltet die symptomatische Trigeminusneuralgie in Folge einer Multiplen Sklerose und die Diagnose Nr. 6 die postzosterische Neuralgie. Die letze Diagnosegruppe Nr. 7 soll demnach die atypischen Gesichtsschmerzen beinhalten, die durch eine somatoforme Schmerzstörung ausgelöst werden und psychologische Untersuchungen erfordern.

An diesen zwei Beispielen wird die Komplexität der Thematik ,neuralgischer Trigeminusschmerz" deutlich. Wenn im weiteren Verlauf dieser Arbeit von einer Trigeminusneuralgie $(\mathrm{TN})$ oder von Trigeminusneuralgiepatienten (TN-Patienten) ohne weitere Spezifizierung die Rede ist, bezieht sich dies auf die klassische Trigeminusneuralgie.

\section{Bildgebende Verfahren}

Die Anwendung bildgebender Verfahren kann unter Umständen hilfreich sein, die klassische von der symptomatischen TN zu unterscheiden. Mittel der Wahl ist hierbei die Magnetresonanztomographie (MRT), welche in bis zu $15 \%$ der Fälle strukturelle Läsionen identifizieren kann (Gronseth et al. 2008). Daher wird die MRT nicht nur in der Literatur als mögliches Diagnostikum empfohlen (Goru und Pemberton 2009, Zacest et al. 2010), sondern bereits in der UMG routinemäßig vor jeder Operation einer klassischen TN zum Ausschluss einer symptomatischen TN angewendet (als Beispiel siehe Abbildung 1.2). 
Abbildung 1.2: MRT bei Gefäßkompression des N. trigeminus rechts

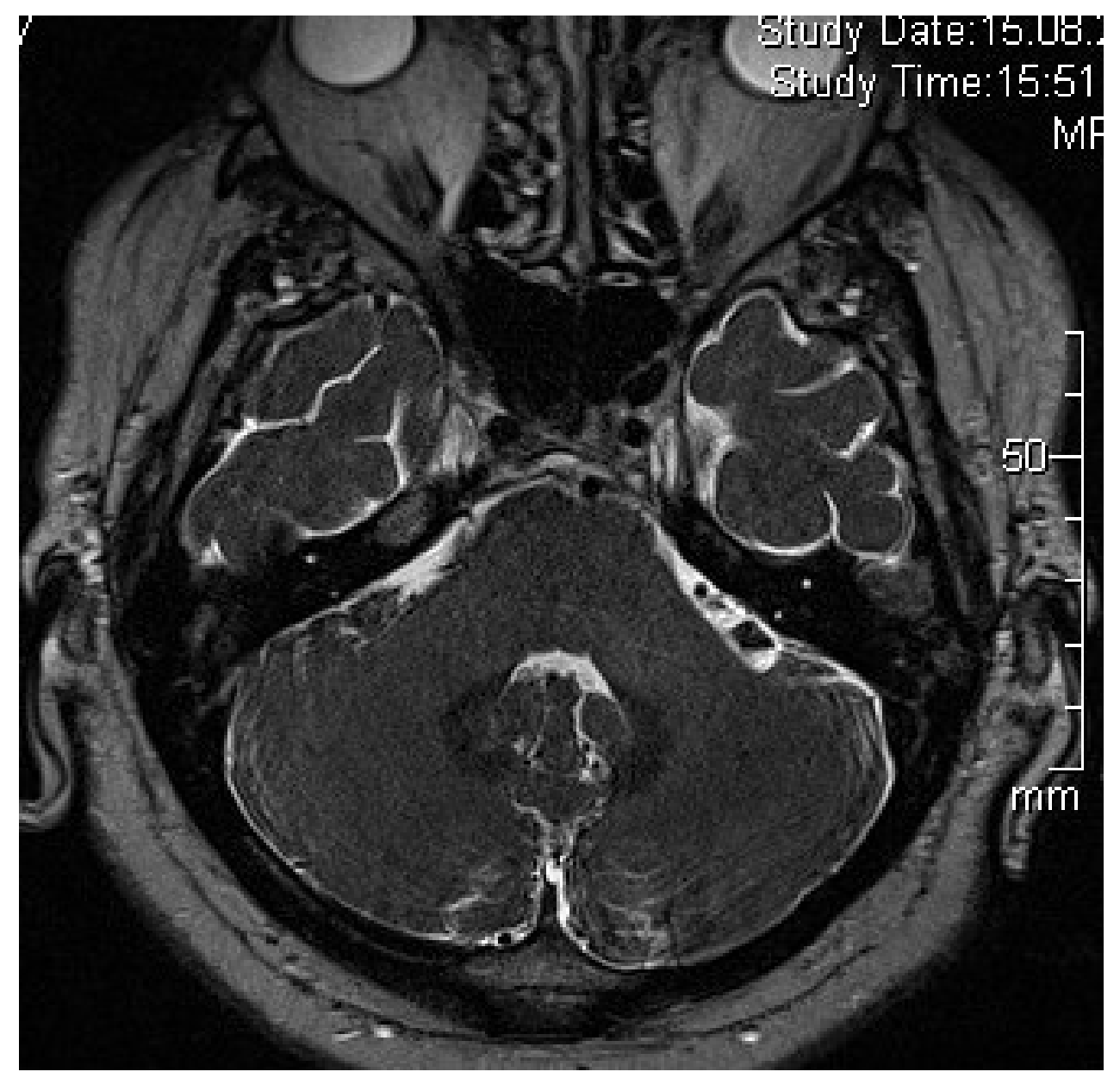

\subsubsection{Therapieformen}

Wurde eine klassische TN diagnostiziert, wird allseits die medikamentöse Therapie als erstes therapeutisches Mittel angesehen und angewendet (Cruccu et al. 2008, Lemos et al. 2011, Nurmikko und Eldridge 2001, Zakrzewska 2001, Zakrzewska et al. 2012). Bei symptomatischen TN ist in der Regel die Therapie der entsprechenden Grunderkrankung angezeigt.

\section{Medikamentöse Therapie}

Das Mittel der Wahl ist derzeit das Antikonvulsivum Carbamazepin, welches chemisch mit den trizyklischen Antidepressiva verwandt ist. Es wird vermutet, dass das Medikament seine Wirkung über die Blockade von Natriumkanälen an der Nervenmembran entfaltet, was die Weitergabe ektopischer Signale verhindert (Backonja 2003, Cruccu et al. 2008, Krafft 2008, Taylor et al. 1981, Zakrzewska 2001). Der genaue Wirkmechanismus ist trotz einer Mehrzahl von Untersuchungen (Kawaura et al. 2011, Siniscalchi et al. 2011) noch nicht abschließend geklärt. 
Alternativ können auch die Medikamente Oxcarbacepin, Gabapentin oder Lamotrigin sowie zur Behandlung von Schmerzattacken Phenytoin oder Baclofen eingesetzt werden (Stienen et al. 2010, Zakrzewska 2010 a, 2010 b).

Oxcarbazepin ist ein Abkömmling des Carbamazepins und soll geringere Nebenwirkungen aufweisen, allerdings ist ist es noch nicht ausführlich genug erprobt und beschrieben worden. Gabapentin zeigt erste ansprechende Ergebnisse, es fehlen aber ebenfalls ausreichende Untersuchungen (Cole et al. 2005, Sommer 2002). Lamotrigin muss sehr langsam titriert verabreicht werden, weil es sonst zu gefährlichen allergischen Hautund Schleimhautreaktionen kommen kann. Daher wird es zur Zeit eher als sogenanntes add-on-Präparat zusammen mit Carbamazepin verwendet (Backonja 2003, Canavero und Bonicalzi 1997).

Phenytoin kam über viele Jahre zum Einsatz und ist das erste Medikament mit guten Ergebnissen gewesen. Obwohl bis heute keine randomisierten, doppel-blinden Studien vorliegen, wird es auch heute noch vor allem im Bereich der Akuttherapie eingesetzt (Cheshire 2001, Cruccu et al. 2008, Stienen et al. 2010). Baclofen zeigt eine Wirksamkeit bei bis zu $74 \%$ der Patienten und wird in manchen Kliniken auch nach erfolgloser Einzelgabe von Carbamazepin als Mittel der zweiten Wahl in Kombination mit diesem verabreicht (Fromm et al. 1984, Krafft 2008).

\section{Chirurgische Therapie}

Zeigen medikamentöse Verfahren keine ausreichende Wirkung oder übersteigen die auftretenden Nebenwirkungen den schmerzhemmenden Effekt, stehen mehrere chirurgische Techniken zur Wahl. Bei etwa der Hälfte der erkrankten Personen kommt es im Laufe der Erkrankung zu chirurgischen Eingriffen (Barker et al. 1996, Ibrahim 2012), die nach Zakrzewska (2010 a) anhand der OP-Region in drei Kategorien eingeteilt werden:

\section{Posteriore Fossa}

2. Ganglion Gasseri-Bereich

\section{Periphere Techniken}

Bevor genauer auf die Techniken eingegangen wird, gilt allgemein festzuhalten, dass die Prognose eines chirurgischen Eingriffs durch unterschiedliche Faktoren beeinflusst werden kann. Für gewöhnlich bedeutet zum Beispiel ein geringeres Alter ein verringertes Risiko für Komplikationen. Für TN-Patienten konnte zusätzlich festgestellt werden, dass eine lange präoperative Leidenszeit die Prognose für eine dauerhafte postoperative Schmerzfreiheit verschlechtern kann (Barker et al. 1996, Cohen 2005, Sarsam et al. 2010, Toda 2008). 


\section{Posteriore Fossa}

Die in der posterioren Fossa durchgeführte und heutzutage am häufigsten angewandte Methode ist die mikrovaskuläre Dekompression (MVD). Bei dieser Operation wird über eine retromastoidale Kraniektomie der Zugang zum Kleinhirnbrückenwinkel geschaffen, der Trigeminusnerv identifiziert und von arachnoidalen Verwachsungen befreit. Anschließend wird der pathologische Gefäß-Nerven-Kontakt lokalisiert und beide Strukturen durch die Einlage eines Interponats, zum Beispiel aus Teflon, separiert (Broggi et al. 2000, Jannetta 1980, McLaughlin et al. 1999, Nurmikko und Eldridge 2001, Stienen et al. 2010).

Somit ist die MVD nicht nur die einzige nicht-destruktive Methode, sondern weist mit $71 \%$ Schmerzfreiheit nach 10 Jahren ohne Medikation die besten Langzeiterfolge aller chirurgischen Methoden auf (Barker et al. 1996, Sarsam et al. 2010, Stienen et al. 2010, Tronnier et al. 2001, Zakrzewska 2010 a, Zakrzewska und Coakham 2012). Schon 1980 konnte Jannetta bei 79,8 \% von 411 operierten Patienten eine initiale Schmerzfreiheit erreichen, die mithilfe weiterer Operationen auf 83,5\% gesteigert werden konnte. Heute kann eine initiale Schmerzfreiheit bei 95-99 \% der Patienten realisiert werden (Cole et al. 2005). Die wichtigsten Langzeitkomplikationen sind mit bis zu $7 \%$ Ausfälle der Sensibilität, mit 4\% schwere Komplikationen wie Liqourlecks oder Infarkte und mit 1-2 \% der ipsilaterale Hörverlust (Barker et al. 1996, 1997, McLaughlin et al. 1999, Stienen et al. 2010, Zakrzewska 2010 a). Die Mortalität liegt bei lediglich 0,2 - 0,4 \% (Degn und Brennum 2010, Kalkanis et al. 2003, Zakrzewska 2010 a).

Eine weitere Technik, die in der posterioren Fossa zur Anwendung kommt, ist die stereotaktische Radiochirurgie mit dem sogenannten „Gamma Knife“. Hierbei wird unter MRT-Kontrolle mit Gammastrahlen aus 201 Cobalt-60-Quellen das betroffene Areal mit einer Dosis von 70-85 Gy bestrahlt (Kondziolka et al. 2010, Nurmikko und Eldridge 2001, Pagni et al. 2008). Es handelt sich somit zwar um eine nicht-invasive, aber dennoch ablative Technik, welche sich noch auf lange Sicht beweisen muss. In den wenigen vorhandenen Studien wird eine komplette Schmerzfreiheit nur bei durchschnittlich $53 \%$ der Patienten nach 3 Jahren erreicht, wobei diese in einem Zeitraum von 2 Wochen bis zu 2,7 Monaten einsetzen kann (Gronseth et al. 2008, Toda 2008). Obwohl bessere Ergebnisse ohne chirurgische Vorgeschichte erzielt werden, wird die Technik sowohl nach erfolglosen vorrangegangenen Therapien als auch bei älteren und multimorbiden Patienten eingesetzt (Kondziolka et al. 2010, Pagni et al. 2008, Toda 2008). Dies ist auf die Nicht-Invasivität und die geringe Komplikationsrate zurückzuführen. Die Mortalität liegt folglich bei null. Zu den Komplikationen zählen der Hörverlust und Dysästhesien. 


\section{Ganglion Gasseri-Bereich}

In Höhe des Ganglions Gasseri werden drei Techniken beschrieben, denen gemein ist, dass sie ablativer Natur sind und jeweils mittels eines perkutanen Zugangs eine Kanüle durch das Foramen ovale in den Bereich des Ganglions vorgeschoben wird. Allen Methoden ist ebenfalls gleich, dass nach ca. 4 Jahren bei $50 \%$ der Patienten das schmerzfreie Intervall endet und die Mortalität nahezu gleich null ist (Zakrzewska 2010 a).

Bei der Radiofrequenzthermokoagulation oder Radiofrequenzgangliolyse wird noch heute, ähnlich wie von Sweet und Wepsic (1974) beschrieben, durch eine Kanüle eine Radiofrequenzelektrode eingeführt und das Ganglion wiederholt 45-90 Sekunden lang bei $60-90^{\circ} \mathrm{C}$ läsioniert (siehe Abblidungen 1.3 und 1.4). Dabei soll der Patient vor der Läsionierung aufgeweckt werden und durch Stimulation die Position der Sonde im Bereich des zu behandelnden Nervenastes kontrolliert werden. Bei dieser Technik ist mit einer initialen Schmerzfreiheit von 96-99 \% zu rechnen (Lopez et al. 2004, Pagni et al. 2008). Sie führt allerdings auch bei 25-27\% zu mitunter sehr schmerzhaften Dysästhesien (Nurmikko und Eldridge 2001, Pagni et al. 2008). Andere Komplikationen können die Anaesthesia dolorosa oder eine Keratitis sein.

Abbildung 1.3: Lage der Kanüle bei Gangliolyse (von lateral)

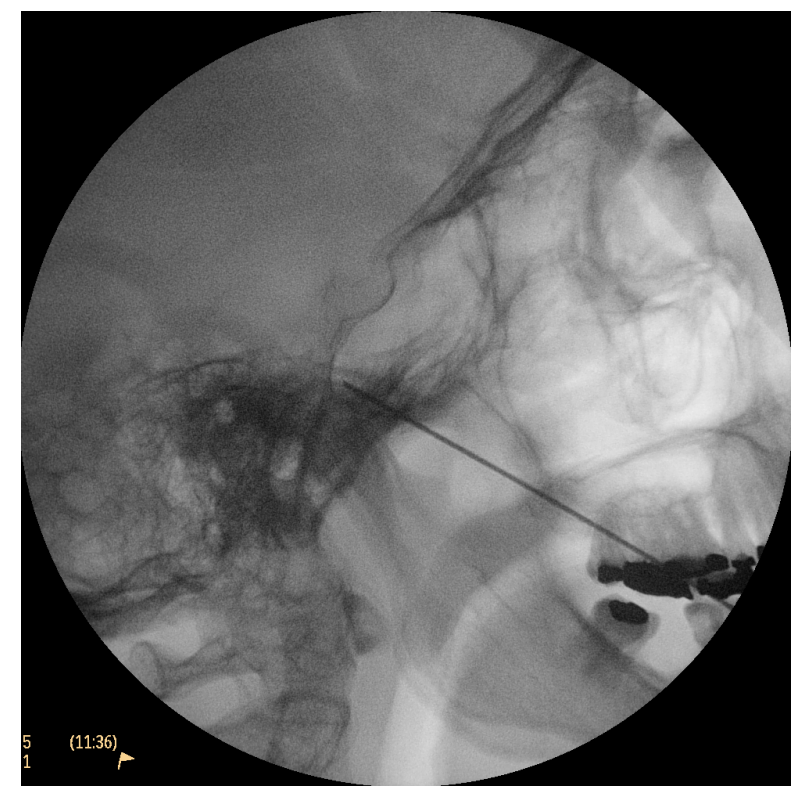


Abbildung 1.4: Lage der Kanüle bei Gangliolyse (Schrägaufnahme)

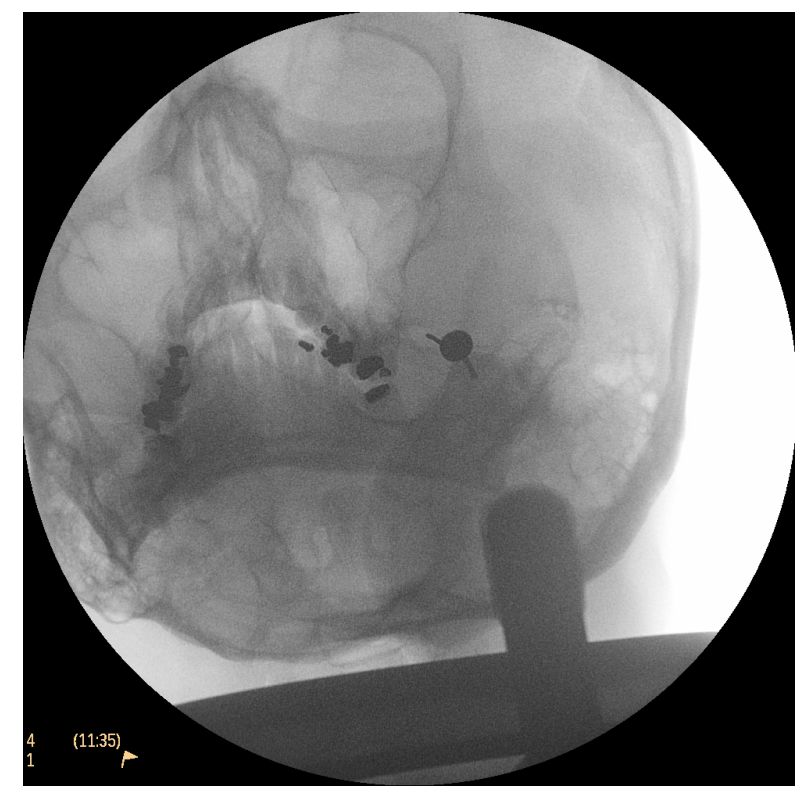

Eine weitere Technik, die am Ganglion Gasseri ansetzt, ist die Glycerinrhizotomie oder Glycerol Gangliolyse. Hierbei wird Glycerol über die Kanüle in das Cavum Meckeli injiziert, wobei der wache bis leicht sedierte Patient oftmals ein Kratzen oder Brennen im affektierten Ast verspürt. Die Gangliolyse führt in 80-90 \% der Fälle zur initialen Schmerzfreiheit (Degn und Brennum 2010, Toda 2008) und es konnte gezeigt werden, dass der Grad des postoperativen Sensibilitätsverlustes mit der Länge des schmerzfreien Intervalls korreliert (Bergenheim et al. 1991). Diese Methode gilt als sicher und kostengünstig durchführbar.

Die dritte Technik ist die Ballonkompression, bei der ein Fogarty-Ballonkatheter in allgemeiner Anästhesie in das Cavum Meckeli vorgeschoben und über einen Zeitraum von 1-6 Minuten mit einem Kontrastmittel gefüllt wird. Dadurch werden die umgebenen Strukturen komprimiert (Nurmikko und Eldridge 2001, Stienen et al. 2010, Toda 2008). Diese Methode führt ebenfalls zu einer initialen Schmerzfreiheit von über $90 \%$ (Bergenheim et al. 2012, Toda 2008). Die Komplikationen ähneln dabei den vorher genannten Methoden mit der Besonderheit, dass in bis zu 50 \% der Fälle von seltenen chronischen Kaubeschwerden berichtet wird (Stienen et al. 2010).

\section{Periphere Techniken}

Periphere Techniken erzielen die Blockade oder Destruktion von Trigeminusteilen distal des Ganglion Gasseri. Sie kommen beispielsweise zum Einsatz, wenn eine schnelle Schmerzausschaltung erreicht werden soll, um dem Patienten eine rationale Entscheidung über das weitere chirurgische Vorgehen zu ermöglichen oder in Gebieten auf der Welt, in denen keine entsprechend ausgestatteten Zentren erreichbar sind (Ali et al. 
2012). Zu nennen sind die Kryotherapie, Neurektomie, periphere Alkohol- oder Lidocaininjektion, Akupunktur oder die Gabe von Streptomycin. Bei diesen Methoden sind im Durchschnitt nur $22 \%$ der Patienten nach 2 Jahren noch schmerzfrei, jedoch sind die Komplikationsraten im Vergleich zu den vorher genannten Methoden relativ gering (Toda 2008, Zakrzewska 2010 a).

\subsection{Fragestellung}

Mit dem Wissen über die TN und besonders unter dem Gesichtspunkt, dass die Schmerzsensation von TN-Patienten zu über $\frac{4}{5}$ in den Ausbreitungsgebieten des N. maxillaris und des N. mandibularis angegeben wird (Katusic et al. 1990, Merrill und GraffRadford 1992) und sowohl Kauen als auch Zähneputzen und Berührungen in der perioralen Region als Trigger angegeben werden, lässt sich die Hypothese aufstellen, dass viele Patienten einen odontogenen Auslöser für ihre Beschwerden in Betracht ziehen. Die Vorstellung beim Zahnarzt als initialer Arztkontakt wäre damit die logische Konsequenz. Sollte dies der Fall sein und berücksichtigt man, dass es sich bei der TN um eine seltene Krankheit handelt, besteht die Möglichkeit, dass sie als solche vom Zahnarzt nicht diagnostiziert wird oder sogar eine inadäquate Therapie durchgeführt wird.

Es gilt also herauszufinden, ob Patienten mit einer TN auf Grund ihrer Schmerzsymptomatik als erstes einen Zahnarzt aufsuchen und wenn ja, wie sich der weitere Behandlungsablauf in der zahnärztlichen Praxis gestaltet.

Diese Hypothese ist bereits von einigen Autoren, die sich mit dem Thema TN beschäftigen (Horowitz et al. 2004, Israel und Scrivani 2000, Shankland 2001, Truelove 2004, Zakrzewska 2002, 2010 a), aufgestellt worden, wurde aber kaum genauer untersucht. Sollte sich die Hypothese bestätigen und sich der Zahnarzt als Erstkontakt herauskristallisieren, lohnt es sich ebenfalls genauer zu untersuchen, bei wie vielen Patienten der Zahnarzt bereits eine Verdachtsdiagnose stellen konnte, wie mögliche zahnärztliche Therapieansätze aussahen, wann der Patient wohin überwiesen wurde, und wie viel Zeit bis zu der endgültigen „Diagnose Trigeminusneuralgie“ verging.

Des Weiteren ergeben sich Fragen danach, wie stark das Thema respektive die Problematik TN in der Zahnärzteschaft bekannt ist. Was unternehmen Zahnärzte, wenn sie in ihrer Praxis die Verdachtsdiagnose TN stellen? Wie häufig kommt das vor? Was wissen Zahnärzte von der Trigeminusneuralgie? Und wie schätzen die Zahnärzte selbst ihre Fähigkeiten ein?

Zur Beantwortung dieser Fragen und um eine möglichst differenzierte Sicht zu erhalten erscheinen folglich zwei Personenkreise als geeignet: zum einen Patienten mit einer diagnostizierten TN und zum anderen praktizierende Zahnärzte. 


\section{Material und Methoden}

Für diese retrospektive Studie sollen Patienten befragt werden, die im Zeitraum vom 1. Januar 2003 bis zum 31. Dezember 2008 in der Neurochirurgischen Klinik und Poliklinik der Universitätsmedizin Göttingen (UMG) auf Grund der Diagnose Trigeminusneuralgie operiert worden sind. Es galt einen Fragebogen zu entwickeln und damit ein standardisiertes telefonisches Interview durchzuführen. Dieses Vorhaben ist im ersten Schritt der Ethik-Kommission der Georg-August-Universität Göttingen vorgelegt und am 01.07.2009 ohne Bedenken bewilligt worden.

Weiterhin sollen alle im Stadtbezirk Göttingen niedergelassenen Zahnärzte angeschrieben und zu dem Thema Trigeminusneuralgie mit Hilfe eines ebenfalls selbst konzipierten Fragebogens befragt werden.

\subsection{Anschreiben}

Für die Studie sind zwei Anschreiben entworfen worden - das Anschreiben Patienten und das Anschreiben Zahnärzte (s. Anhang).

Das Anschreiben Patienten beinhaltete eine kurze Vorstellung der geplanten Studie und umseitig die Bitte zur Einwilligung in eine telefonischen Kontaktaufnahme durch den Doktoranden. Jedem Anschreiben war ein bereits frankierter, mit Adressaufkleber der Klinik für Neurochirurgie Göttingen versehener Rückumschlag beigelegt.

Das Anschreiben Zahnärzte enthielt ebenfalls eine kurze Vorstellung der geplanten Studie und auf der Rückseite einen aus sieben Fragen bestehenden Fragebogen. Jedem Anschreiben war wiederum ein bereits frankierter, mit Adressaufkleber der Klinik für Neurochirurgie Göttingen versehener Rückumschlag beigelegt.

\subsection{Trigeminusneuralgiepatienten}

Die Suche in der Datenbank der UMG mit Hilfe der elektronischen OP-Dokumentation (Siemens Medico//S) ergab, dass im Zeitraum von Januar 2003 bis Dezember 2008 insgesamt 118 Operationen an 92 TN-Patienten in der Neurochirurgie durchgeführt wurden. Diese teilten sich auf in 82 perkutane Rhizotomien, 33 mikrovaskuläre De- 
kompressionen und 3 mal Motorkortexstimulationen als schmerztherapeutische Maßnahmen. Im Folgenden wurde 92 TN-Patienten das Anschreiben Patienten zugeschickt und mit 57 Antwortschreiben konnte eine Rücklaufquote von $62 \%$ erreicht werden.

Abhängig vom angewendeten Operationsverfahren wurden die Patienten einer von drei OP-Gruppen zugeordnet. In die OP-Gruppe I wurden Patienten eingeteilt, bei denen eine perkutane Destruktion des Ganglions Gasseri durchgeführt worden war. Die OP-Gruppe II beinhaltet Patienten, bei welchen eine mikrovaskuläre Dekompression durchgeführt worden war und die OP-Gruppe III enthält die Patienten, bei denen beide Verfahren zum Einsatz gekommen sind. Folglich konnten 32 Patienten der OP-Gruppe I, 17 der OP-Gruppe II und 2 der OP-Gruppe III zugeordnet werden. Auf Grund der sehr geringen wissenschaftlichen Aussagekraft wurde die aus 2 Personen bestehende OP-Gruppe III aufgelöst und die Personen entsprechend ihrer ersten Operation der OP-Gruppe I zugeordnet. Demzufolge setzt sich diese nun aus 34 Patienten zusammen.

\subsection{Zahnärzte}

Mit dem Anschreiben Zahnärzte wurden alle zum Zeitpunkt der Studie (18.09.2009) niedergelassenen Zahnärzte in Göttingen angeschrieben, die auf der Internetseite der Deutschen Telekom Medien GmbH, www.dastelefonbuch.de, unter der Suchbegriffkombination Zahnarzt und Göttingen verzeichnet waren. Es konnten 87 Praxen identifiziert werden, von denen 11 als Gemeinschaftspraxen auftraten. In diesen Fällen wurden dem Anschreiben zwei Fragebögen beigelegt. Demzufolge wurden 98 Zahnärzte angeschrieben.

\subsection{Fragebögen}

Fragebögen dienen der zweckgerichteten Datenerhebung und ermöglichen durch die Zusammenstellungen strukturierter Fragen, Einhaltung formaler Regeln und Verwendung erprobter Techniken unter anderem eine deskriptive statistische Auswertung. Man unterscheidet geschlossene Fragen von halb offenen und offenen Fragen. Bei geschlossenen Fragen ist die Antwortkategorie vorgegeben, wie zum Beispiel bei einer Ja/Nein-Frage. Offenen Fragen hingegen werden durch ein Fragewort eingeleitet und der Befragte kann seine Antwort frei formulieren. Halb offene Fragen, auch Hybridfragen genannt, geben zwar keine definierte Antwortkategorie vor, lassen jedoch eine Antwort aus ein bis zwei Wörtern erwarten.

Bei dieser Studie kamen zwei Individual-Fragebögen zum Einsatz, mit denen einzelne Personen zu Ihrem persönlichen Kenntnisstand und Einschätzungen befragt wurden. Bei diesen Fragebögen handelt es sich im ersten Fall um einen als Fragebogen auf- 
gebauten Leitfaden für ein strukturiertes telefonisches Interview mit den Patienten (s. Anhang), im Folgenden Fragebogen Patienten genannt. Der zweite Fragebogen, genannt Fragebogen Zahnärzte (s. Anhang), befand sich auf der Rückseite des Anschreibens Zahnärzte und dient dem selbstständigen Ausfüllen seitens der Zahnärzte. Hierbei diente ein Fragebogen von Pemberton et al. (2001) als Orientierung.

Alle in den zwei Fragebögen vorkommenden geschlossenen Fragen sind nominalskaliert, da sie lediglich qualitativ-beschreibend sind und in keine Rangfolge gebracht werden können.

\subsubsection{Fragebogen Patienten}

Der Fragebogen Patienten besteht aus insgesamt 9 Fragen, die sich aus sechs geschlossenen Fragen, die mit Ja oder Nein beantwortet werden können (die Fragen Nr. 1, 2, 2a, 2b, 2c und 3) (Nominalskala), zwei halb offenen (Nr. 2ci und Nr. 2d) und einer offenen Frage (Nr. 2bi) zusammensetzten. Aufgebaut ist der Fragebogen aus den drei Hauptfragen Nr. 1, Nr. 2 und Nr. 3, wobei die Frage Nr. 2 eine Filterfunktion erfüllt. Der folgenden Fragenblock mit den Unterfragen Nr. 2a, 2b, 2bi, 2c, 2ci und 2d bezieht sich auf das Ereignis eines Zahnarztbesuches. Die Beantwortung der Hauptfrage Nr. 2 mit Nein führte zum Überspringen aller Unterfragen. In dem Fall ist dem Patienten nur noch die Frage Nr. 3 gestellt worden. Die Fragen Nr. 2bi und Nr. 2ci sind ebenfalls nur den Personen gestellt worden, wenn die vorangegangene Frage mit Ja beantwortet wurde. Das Auftreten eines solchen Filters bzw. einer solchen Gabelung wird auf dem Fragebogen durch jeweiliges Einrücken, die Einführung einer Unterkategorie sowie ein der Frage vorangestelltes wenn ja deutlich gemacht.

Die Fragen Nr. 1 und Nr. 2 bilden die Kategorie Initialer Arztkontakt.

- Die Frage Nr. 1 diente als sogenannte „Eisbrecher-Frage“. Zur Orientierung über das allgemeine Verhältnis des Patienten zum Zahnarzt soll erfragt werden, ob der Patient vor dem Auftreten der Trigeminusneuralgie regelmäßig in zahnärzlicher Behandlung war. Die Frage Nr. 1 lautet demnach: Waren sie im Vorfeld Ihrer Trigeminusneuralgiebehandlung im Göttinger Universitätsklinikum in regelmäßiger zahnärztlicher Behandlung? Im Falle einer Nachfrage zur Bedeutung von „regelmäßig“ wurde im telefonischen Interview auf die jährliche Vorsorgeuntersuchung verwiesen.

- Um herauszufinden, ob Trigeminusneuralgiepatienten bei dem Auftreten von Beschwerden bzw. Schmerzen im Gesichtsbereich als erstes zum Zahnarzt gehen, wird in der Frage Nr. 2 gefragt: Sind Sie auf Grund Ihrer damaligen Beschwerden/Schmerzen als erstes zum Zahnarzt gegangen?

Die Fragen Nr. 2a bis Nr. 3 fallen in die Kategorie Zahnärzliche Evaluation und Maßnahmen. 
- Für den Fall, dass der Patient/die Patientin die Frage 2 mit Ja beantwortet und demnach als erstes zum Zahnarzt ging, interessierte, ob der Zahnarzt zu einem unbestimmten Zeitpunkt in der darauf folgenden Behandlungszeit die Verdachtsdiagnose Trigeminusneuralgie geäußert hat. Die Frage Nr. 2a lautet: wenn ja: Hat Ihr Zahnarzt die Diagnose Trigeminusneuralgie stellen können?

- Die Frage Nr. 2b soll aufzeigen, ob die Zahnärzte nach Abschluss Ihrer Diagnostik eine Therapie auf Grund der vorliegenden Beschwerdesymptomatik durchgeführt haben: Wurde vom Zahnarzt eine Behandlung durchgeführt?

- Sollte der Patient die vorangegangene Frage mit Ja beantworteten, wird in der Frage Nr. 2bi der Ablauf der anschließenden Behandlung erfragt: wenn ja: Wie gestaltete sich der weitergehende Behandlungsverlauf?

- Die Frage Nr. 2c soll herausstellen, ob der Zahnarzt zur Klärung der Sachlage eine Überweisung vorgenommen hat und lautet: Hat der Zahnarzt eine Überweisung vorgenommen?

- Für den Fall, dass der Zahnarzt eine Überweisung vorgenommen hat, wird gefragt (Frage Nr. 2ci): wenn ja: An wen hat Sie Ihr Zahnarzt überwiesen?

- Die Frage Nr. 2d soll Aufschluss darüber geben, wie sich der zeitliche Verlauf zwischen dem Auftreten der ersten Symptome, dem Zahnarztbesuch und der Inanspruchnahme einer neurochirurgischen/ neurologischen Begutachtung gestaltet hat. Die Frage lautet: Können Sie sagen, wie viel Zeit zwischen (1.) den ersten Beschwerden, (2.) dem Zahnarztbesuch und (3.) einer neurochirurgischen Begutachtung vergangen ist?

- Die Frage Nr. 3 ist wiederum eine Frage, die jedem Patienten gestellt wurde: Haben Sie momentan Beschwerden?

Es muss darauf hingewiesen werden, dass die Fragen nicht in jedem Interview wörtlich der Vorlage entsprechend formuliert worden sind, sondern je nach Verlauf des Gespräches dem Kontext entsprechend eingebunden wurden. Die Reihenfolge wurde immer beibehalten.

\subsubsection{Fragebogen Zahnärzte}

Der Fragebogen Zahnärzte besteht aus sieben geschlossenen Fragen, wobei die Fragen 1 und 2 mit der Angabe eines Zahlenwertes beantwortet werden können.

- Mit Hilfe der Frage 1 soll herausgefunden werden, wie häufig Göttinger Zahnärzte in einem bestimmten Zeitraum die Verdachtsdiagnose Trigeminusneuralgie ausgesprochen haben. Es soll sich hierbei in etwa um den bei der Auswahl des Patientenkollektivs verwendeten Zeitraum (Januar 2003 bis Dezember 2008) handeln. Die Frage heißt wie folgt: Wie viele Male in den letzten sechs Jahren 
konnte von Ihnen schätzungsweise die Verdachtsdiagnose "Trigeminusneuralgie“ gestellt werden? Es besteht die Möglichkeit einen beliebigen Zahlenwert (1-...) in das abgebildete Feld handschriftlich einzutragen bzw. im Falle der Aussage Null (keinmal) diesen Wert einzutragen oder das Feld zu streichen.

- Die zweite Frage soll einen Überblick verschaffen über das ungefähre Aufkommen von Trigeminusneuralgiepatienten in Göttinger Zahnarztpraxen im betrachteten Zeitraum. Frage 2: Wie viele Patienten mit einer bekannten Trigeminusneuralgie haben Sie in den letzten sechs Jahren behandelt? Es kann wiederum ein Zahlenwert in das Kästchen vor dem Wort Patienten handschriftlich eingetragen werden.

Die Fragen 3 bis 7 können mit Ja oder Nein beantwortet werden indem das zutreffende Feld angekreuzt wird (Nominalskala).

- Die dritte Frage soll der kritischen Selbsteinschätzung des Zahnarztes dienen. Frage 3 lautet demnach: Wie schätzen Sie sich selbst ein: Würden Sie eine Trigeminusneuralgie erkennen?

- Frage 4 dient der Einschätzung des Bekanntheitsgrades des bei Trigeminusneuralgiepatienten am häufigsten verschriebenen Medikamentes Carbamazepin und liest sich folgendermaßen: Ist Ihnen das Medikament Carbamazepin bekannt?

- Frage 5 ziehlt auf die Überprüfung des Wissensstandes des Zahnarztes ab: Sind Ihnen neben medikamentösen auch chirurgische Ansätze der Therapie bekannt?

- Frage 6 wiederum soll aufzeigen, wie die Zahnärzteschaft den derzeitigen Wissensstand Ihres Berufszweigs über das Thema Trigeminusneuralgie einschätzt indem gefragt wird: Denken Sie, dass ein allgemeiner Fortbildungsbedarf zum Thema „Trigeminusneuralgie in der Zahnarztpraxis" besteht?

- Die siebte und letzte Frage dieses Fragebogens soll abklären, ob im Falle einer Fortführung der Studie oder bei Rückfragen die Bereitschaft besteht, für ein Telefonat zur Verfügung zu stehen. Sie lautet: Stehen Sie ggf. für ein kurzes Telefonat zum Thema Trigeminusneuralgie bzw. Rückfragen zur Verfügung? Zudem wird für den Fall der Einwilligung um den Aufdruck eines Praxisstempels gebeten. 


\section{Ergebnisse}

Im Anschluss an die Genehmigung durch die Ethikkommission der Georg-AugustUniversität Göttingen vom 01.07.2009 wurde an 92 Patienten das Anschreiben Patienten und an 98 Zahnärzte das Anschreiben Zahnärzte verschickt.

\subsection{Trigeminusneuralgiepatienten}

\subsubsection{Gesamtgruppe}

Bis zum 15.10.2009 sind in der Neurochirurgie der UMG 57 Antwortschreiben eingegangen. In 6 Briefen berichteten Angehörige, dass der angeschriebene Patient oder die Patientin mittlerweile verstorben waren. Das telefonische Interview mittels des Fragebogens Patienten wurde demnach im Zeitraum vom 12.08.2009 bis zum 18.10.2009 mit 51 Personen durchgeführt. Daraus ergibt sich eine abschließende Rücklaufquote von $55,4 \%$.

Das Patientenkollektiv setzt sich zusammen aus 26 Frauen und 25 Männern mit einem Durchschnittsalter zu Beginn der Befragung (27.08.2009) von 69,3 Jahren. Den Patienten sind, je nach Beantwortung der Frage 2, drei bis sieben Fragen gestellt worden.

\section{Initialer Arztkontakt}

Die Frage Nr. 1 wurde von allen Befragten beantwortet und zeigt, dass 80,4 \% der Patienten angaben, vor dem Auftreten der ersten Symptome der Trigeminusneuralgie regelmäßig zum Zahnarzt gegangen zu sein.

Über $4 / 5(82 \%)$ sind laut Frage Nr. 2 bei dem Auftreten der Symptome als erstes zum Zahnarzt gegangen (vergleiche Tabelle 3.1).

\section{Zahnärztliche Evaluation und Maßnahmen}

Die Frage Nr. 2a zeigte auf, dass die Verdachtsdiagnose Trigeminusneuralgie nur bei 2 von 43 Patienten vom Zahnarzt gestellt wurde. Außerdem ist bei $60 \%$ der befragten Patienten vom Zahnarzt eine Behandlung durchgeführt worden (Frage Nr. 2b). 
Des Weiteren hat bei etwa der Hälfte der Patienten $(51,2 \%)$ laut Frage Nr. 2c der Zahnarzt eine Überweisung vorgenommen. $2 / 3$ der Befragten gaben zusätzlich in der Frage Nr. 3 an, keine Beschwerden mehr zu haben.

Tabelle 3.1: Ja/Nein-Fragen Fragebogen Patienten (gesamt)

\begin{tabular}{|c|c|c||c|c||c|c|}
\hline Nr. & Frage in Kurzform & {$[\mathrm{n}]$} & Ja & $\%$ & Nein & $\%$ \\
\hline \hline 1 & Waren Sie regelmäßig beim ZA? & 51 & 41 & 80,4 & 10 & 19,6 \\
\hline 2 & Sind Sie als erstes zum ZA gegangen? & 50 & 41 & 82 & 9 & 18 \\
\hline $2 \mathrm{a}$ & Hat Ihr ZA die Verdachtsdiagnose TN stellen können? & 43 & 2 & 4,7 & 41 & 95,3 \\
\hline $2 \mathrm{~b}$ & Wurde vom ZA eine Behandlung durchgeführt? & 45 & 27 & 60 & 18 & 40 \\
\hline $2 \mathrm{c}$ & Hat der ZA eine Überweisung vorgenommen? & 41 & 21 & 51,2 & 20 & 48,8 \\
\hline 3 & Haben Sie momentan Beschwerden? & 50 & 17 & 34 & 33 & 66 \\
\hline
\end{tabular}

$\mathrm{ZA}=$ Zahnarzt

$\mathrm{TN}=$ Trigeminusneuralgie

Die Fragen, die nicht mit Ja oder Nein beantwortet werden konnten, waren die offenen Fragen Nr. 2bi und Nr. 2ci des Interviews. Die Frage Nr. 2bi (Abbildung 3.1) zeigt, dass bei 51 Patienten insgesamt 54 Zähne extrahiert worden sind, 13 Wurzelkanalbehandlungen durchgeführt, 7 Zähne als Pfeiler zur Aufnahme von Zahnersatz beschliffen, 3 Füllungen gelegt und 4 Implantate gesetzt worden sind. Des Weiteren sind bei 4 Patienten mehrfach vom Zahnarzt Injektionen im Ausbreitungsgebiet des N. trigeminus gesetzt worden. 
Abbildung 3.1: Therapiemaßnahmen der Zahnärzte (WBH = Wurzelkanalbehandlung)

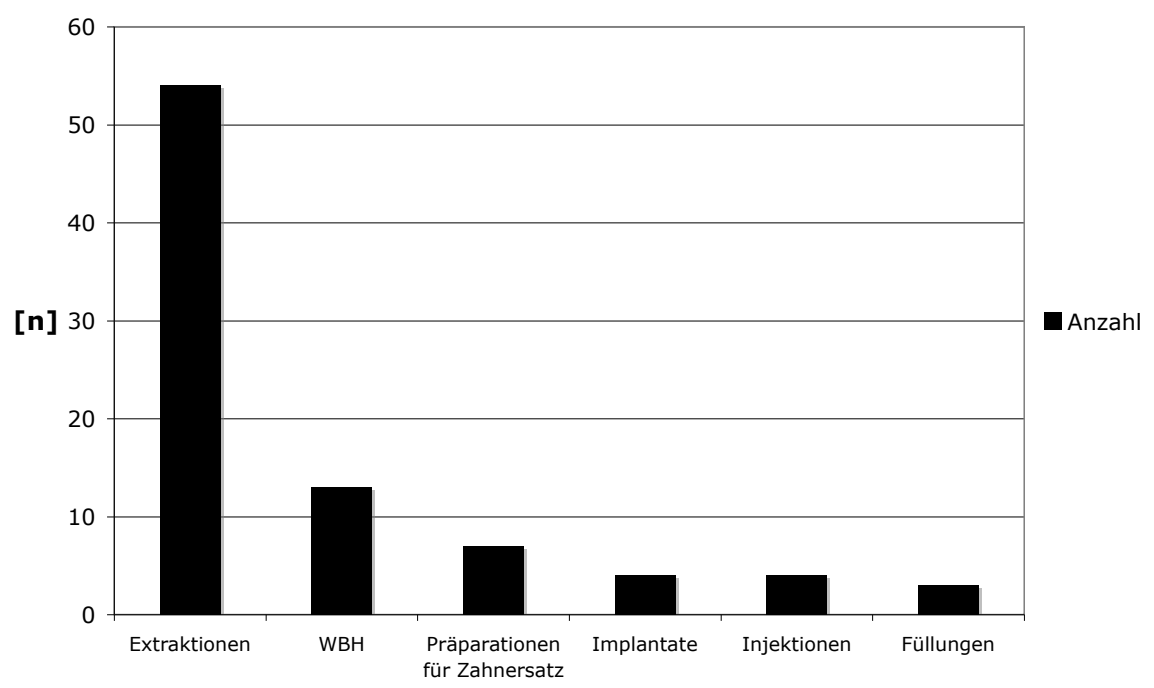

Betrachtet man die Verteilung der insgesamt 54 durchgeführten Extraktionen (Abbildung 3.2) auf die behandelten Patienten, wird ersichtlich, dass über die Hälfte (54,6\%) der registrierten Extraktionen bei zwei Patienten durchgeführt wurden. Der Median $m$ der Extraktionen beträgt 2.

Abbildung 3.2: Verteilung der durchgeführten Extraktionen

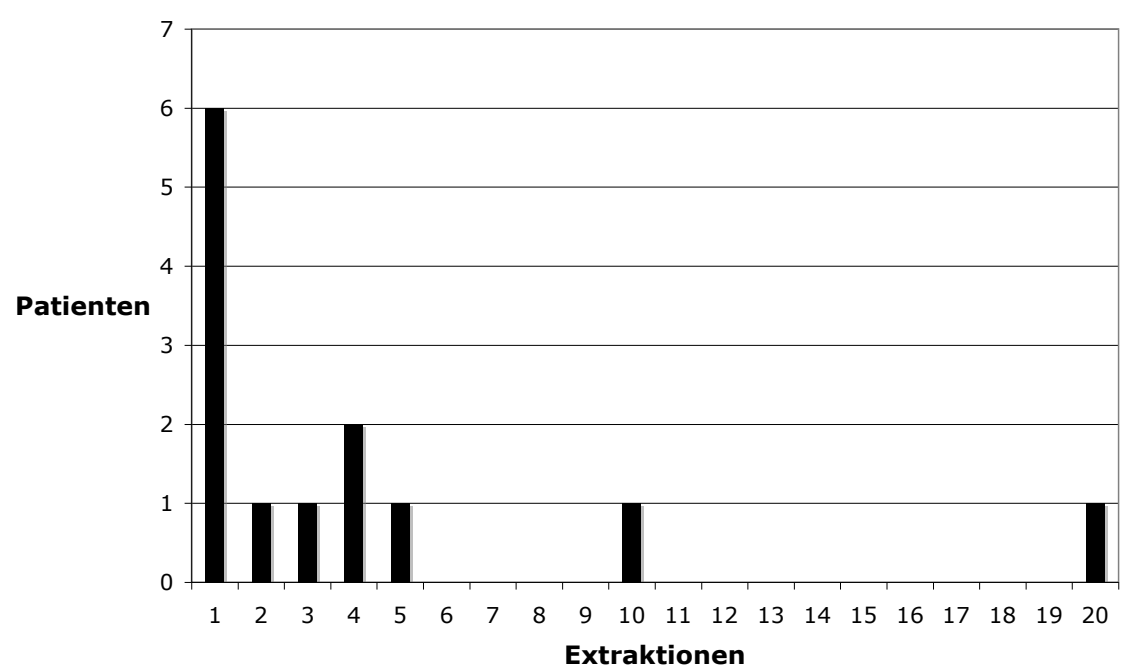


Wie weiterhin aus Tabelle 3.1 hervorgeht, hat bei $51,2 \%$ der befragten Patienten der Zahnarzt eine Überweisung vorgenommen (Frage Nr. 2c). In der Frage Nr. 2ci wurde dann nach dem Fachgebiet des Arztes gefragt, an den überwiesen worden war.

Die Aussagen wurden in 3 Kategorien eingeteilt:

1. neurologisch - Überweisungen an neurologische oder neurochirurgische Einrichtungen

2. zahnmedizinisch - Überweisungen an primär zahnmedizinisch ausgerichtete Einrichtungen wie andere Zahnärzte, Oralchirurgen oder MKG-Chirurgen

3. andersartig - Überweisungen an den Hausarzt oder Physiotherapeuten

Knapp $30 \%$ wurden in eine neurologische Einrichtung (1.), über $60 \%$ in eine zahnärztliche Einrichtung (2.) und jeweils ein Patient zum Hausarzt bzw. Physiotherapeuten (3.) überwiesen (Tabelle 3.2).

Tabelle 3.2: Überweisungen der Zahnärzte

\begin{tabular}{|c|c|c|c|}
\hline & 1. neurologisch & 2. zahnmedizinisch & 3. andersartig \\
\hline \hline$[\mathrm{n}]$ & 6 & 13 & 2 \\
\hline$\%$ & 28,6 & 61,9 & 9,5 \\
\hline
\end{tabular}

In der Frage Nr. 2d wurde nach den zeitlichen Intervallen zwischen drei Ereignissen gefragt:

- (1.) dem ersten Auftreten der Symptome

- (2.) dem Zahnarztbesuch

- (3.) der neurologischen/neurochirurgischen Begutachtung

Es gab drei Intervallkategorien zu betrachten - das Intervall zwischen dem Auftreten der Symptome und dem Aufsuchen des Zahnarztes $(1 . \rightarrow 2$.), das Intervall zwischen dem Aufsuchen des Zahnarztes und einer neurologischen/neurochirurgischen Begutachtung $(2 . \rightarrow 3$. $)$ und das Intervall zwischen dem Auftreten der ersten Symptome und einer neurologischen/neurochirurgischen Begutachtung $(1 . \rightarrow 3$.).

Nicht alle Patienten konnten zu diesen Punkten genaue Angaben machen, da es sich teilweise um lange zurückliegende Ereignisse handelte. 34 Patienten erinnerten sich, wie viel Zeit im Intervall $1 . \rightarrow$ 2. und 28 Patienten wie viel Zeit im Intervall $2 . \rightarrow 3$. vergangen war. 40 Patienten konnten Angaben dazu machen, wie viel Zeit im Intervall 1. $\rightarrow 3$. verstrichen war.

Die folgenden Tabellen 3.3 bis 3.5 zeigen sowohl in absoluten Zahlen als auch prozentual dargestellt, bei wie vielen Patienten wie viel Zeit zwischen den drei Ereignissen vergangen war. Auffallend ist einerseits, dass über $70 \%$ der Befragten innerhalb eines 
Monats nach Auftreten der Symptome zum Zahnarzt gingen. Mehr als $1_{4}$ (26,4\%) kam andererseits erst nach frühestens einem Jahr zum Zahnarzt.

Tabelle 3.3: Intervall zwischen Auftreten erster Symptome und Zahnarztbesuch $(1 . \rightarrow 2$.)

\begin{tabular}{|c|c|c|c|c|c|c|c|}
\hline Intervall & {$[\mathrm{n}]$} & $<1$ Monat & $<3$ Monate & $<6$ Monate & $<12$ Monate & $<24$ Monate & $>24$ Monate \\
\hline Ereignis $1 . \rightarrow 2$. & 34 & 24 & 1 & 0 & 5 & 3 & 1 \\
\hline \hline$\%$ & & 70,6 & 2,9 & 0 & 14,7 & 8,8 & 2,9 \\
\hline
\end{tabular}

Außerdem hat es bei 46,4\% der Befragten über ein Jahr gedauert, bis sie nach dem ersten Besuch beim Zahnarzt in einer neurologischen/neurochirurgischen Einrichtung untersucht wurden. Bei beinahe einem Drittel $(32,1 \%)$ hat es sogar länger als 2 Jahre gedauert.

Tabelle 3.4: Intervall zwischen Zahnarztbesuch und neurolog./neurochirurg. Begutachtung $(2 . \rightarrow 3$.)

\begin{tabular}{|c|c|c|c|c|c|c|c|}
\hline Intervall & {$[\mathrm{n}]$} & $<1$ Monat & $<3$ Monate & $<6$ Monate & $<12$ Monate & $<24$ Monate & $>24$ Monate \\
\hline \hline Ereignis $2 . \rightarrow 3$. & 28 & 5 & 0 & 1 & 9 & 4 & 9 \\
\hline$\%$ & & 17,9 & 0 & 3,6 & 32,1 & 14,3 & 32,1 \\
\hline
\end{tabular}

Desweiteren lagen bei 42,5\% der Befragten insgesamt zwischen dem Auftreten der ersten Symptome und einer neurologischem/neurochirurgischen Begutachtung mehr als 2 Jahre. Nur 22,5\% fanden innerhalb eines halben Jahres den Weg zu einer solchen Einrichtung.

Tabelle 3.5: Intervall zwischen Auftreten erster Symptome und neurolog./neurochirurg. Begutachtung $(1 . \rightarrow 3$.)

\begin{tabular}{|c|c|c|c|c|c|c|c|}
\hline Intervall & {$[\mathrm{n}]$} & $<1$ Monat & $<3$ Monate & $<6$ Monate & $<12$ Monate & $<24$ Monate & $>24$ Monate \\
\hline \hline Ereignis $1 . \rightarrow 3$. & 40 & 5 & 1 & 3 & 8 & 6 & 17 \\
\hline$\%$ & & 12,5 & 2,5 & 7,5 & 20 & 15 & 42,5 \\
\hline
\end{tabular}

\subsubsection{Untergruppenanalyse}

\section{OP-Gruppe I}

Aufgeteilt nach dem chirurgischen Verfahren in Gruppen, besteht die OP-Gruppe I (Gruppe Rhizotomie) aus 18 Männern und 16 Frauen mit einem Durchschnittsalter von 71,65 Jahren.

Die Fragen Nr. 1 und Nr. 2 der Kategorie Initialer Arztkontakt ergaben, dass 85,3 \% der Patienten dieser Gruppe im Vorfeld ihrer Erkrankung regelmäßig zum Zahnarzt gingen und über 4/5 nach dem Auftreten der Symptome als erstes den Gang zum Zahnarzt wählten. 
Die Fragen der Kategorie Zahnärztliche Evaluation und Maßnahmen zeigten, dass bei keinem der Patienten dieser OP-Gruppe die Verdachtsdiagnose TN seitens des Zahnarztes gestellt wurde. Jedoch ist bei mehr als $60 \%$ der Befragten vom Zahnarzt eine Behandlung durchgeführt worden. Über die Hälfte $(53,6 \%)$ erhielt eine Überweisung.

Tabelle 3.6: Ja/Nein- Fragen Fragebogen Patienten (OP-Gruppe I)

\begin{tabular}{|c|c|c||c|c||c|c|}
\hline Nr. & Frage in Kurzform & {$[\mathrm{n}]$} & Ja & $\%$ & Nein & $\%$ \\
\hline \hline 1 & Waren Sie regelmäßig beim ZA? & 34 & 29 & 85,3 & 5 & 14,7 \\
\hline 2 & Sind Sie als erstes zum ZA gegangen? & 33 & 27 & 81,8 & 6 & 18,2 \\
\hline 2a & Hat Ihr ZA die Verdachtsdiagnose TN stellen können? & 26 & 0 & 0 & 26 & 100 \\
\hline 2b & Wurde vom ZA eine Behandlung durchgeführt? & 28 & 17 & 60,7 & 11 & 39,3 \\
\hline 2c & Hat der ZA eine Überweisung vorgenommen? & 28 & 13 & 46,4 & 15 & 53,6 \\
\hline 3 & Haben Sie momentan Beschwerden? & 33 & 12 & 36,4 & 21 & 63,6 \\
\hline
\end{tabular}

Bei der ersten offenen Frage, der Frage Nr. 2bi konnte festgestellt werden, dass es bei den 34 Patienten in Gruppe I zu 38 Extraktionen, 3 Wurzelkanalbehandlungen, 2 Präparationen von Zähnen zur Aufnahme von Zahnersatz und 3 Injektionstherapien gekommen ist. Vom Zahnarzt ausgesprochene Überweisungen (Frage Nr. 2ci) führten die Patienten zu über $2 / 3$ (69 \%) in eine zahnmedizinische Einrichtung (2.) und in 3 Fällen $(23 \%)$ in eine der Kategorie neurologisch (1.) zuweisbare Einrichtung.

\section{OP-Gruppe II}

Die OP-Gruppe II (Gruppe Jannetta) setzt sich aus 7 Männer und 10 Frauen mit einem Durchschnittsalter von 64,53 Jahren zusammen. Von den 17 Patienten dieser Gruppe sind über $70 \%$ im Vorfeld in regelmäßiger zahnärztlicher Behandlung gewesen und 82,4 \% suchten beim Auftreten der Symptome als erstes einen Zahnarzt auf (Initialer Arztkontakt).

In der Kategorie Zahnärztliche Evaluation und Maßnahmen wurde bei 2 von 17 Patienten die Verdachtsdiagnose TN gestellt, mehr als die Hälfte (58,8 \%) ist vom Zahnarzt behandelt worden. 
Tabelle 3.7: Ja/Nein-Fragen Fragebogen Patienten (OP-Gruppe II)

\begin{tabular}{|c|c|c||c|c||c|c|}
\hline Nr. & Frage in Kurzform & Jn] & Ja & Nein & $\%$ \\
\hline \hline 1 & Waren Sie regelmäßig beim ZA? & 17 & 12 & 70,6 & 5 & 29,4 \\
\hline 2 & Sind Sie als erstes zum ZA gegangen? & 17 & 14 & 82,4 & 3 & 17,6 \\
\hline 2a & Hat Ihr ZA die Verdachtsdiagnose TN stellen können? & 17 & 2 & 11,8 & 15 & 88,2 \\
\hline 2b & Wurde vom ZA eine Behandlung durchgeführt? & 17 & 10 & 58,8 & 7 & 41,2 \\
\hline 2c & Hat der ZA eine Überweisung vorgenommen? & 14 & 8 & 57,1 & 6 & 42,9 \\
\hline 3 & Haben Sie momentan Beschwerden? & 17 & 5 & 29,4 & 12 & 70,6 \\
\hline
\end{tabular}

Die Männer und Frauen dieser OP-Gruppe erfuhren Behandlungen in Form von 16 Extraktionen, 4 Wurzelkanalbehandlungen, 5 Präparationen, 1 Injektionstherapie und 4 Implantationen (Frage Nr. 2bi). Von den 7 Patienten dieser Gruppe, die eine Überweisung erhalten haben, wurden jeweils drei in eine neurologische bzw. zahnärztliche Einrichtung überwiesen, ein Patient wurde zum Hausarzt überwiesen (Frage Nr. 2ci). 


\subsection{Zahnärzte}

Bis zum 18.10.2009 sind in der Neurochirurgie der UMG insgesamt 50 ausgefüllte Fragebögen eingegangen. Das entspricht bei 98 versendeten Fragebögen einer Rücklaufquote von $51 \%$.

In der ersten Frage des Fragebogen Zahnärzte sollten die Zahnärzte einschätzen, wie oft sie in den letzten sechs Jahren (September 2003 bis September 2009) die Verdachtsdiagnose Trigeminusneuralgie gestellt haben (Abbildung 3.3). Dies war bei 49 Zahnärzten, die die Frage beantworteten, 96 mal in diesem Zeitraum der Fall.

Bemerkenswert ist dabei, dass knapp die Hälfte der Befragten (49 \%) im beschriebenen Sechsjahres-Zeitraum nur ein Mal oder gar kein Mal die Verdachtsdiagnose gestellt haben. Dem gegenüber stehen zwei Zahnärzte, die jeweils 10-mal die Verdachtsdiagnose geäußert haben wollen.

Abbildung 3.3: Frage Nr. 1 - Häufigkeit der Verdachtsdiagnose TN pro Zahnarzt

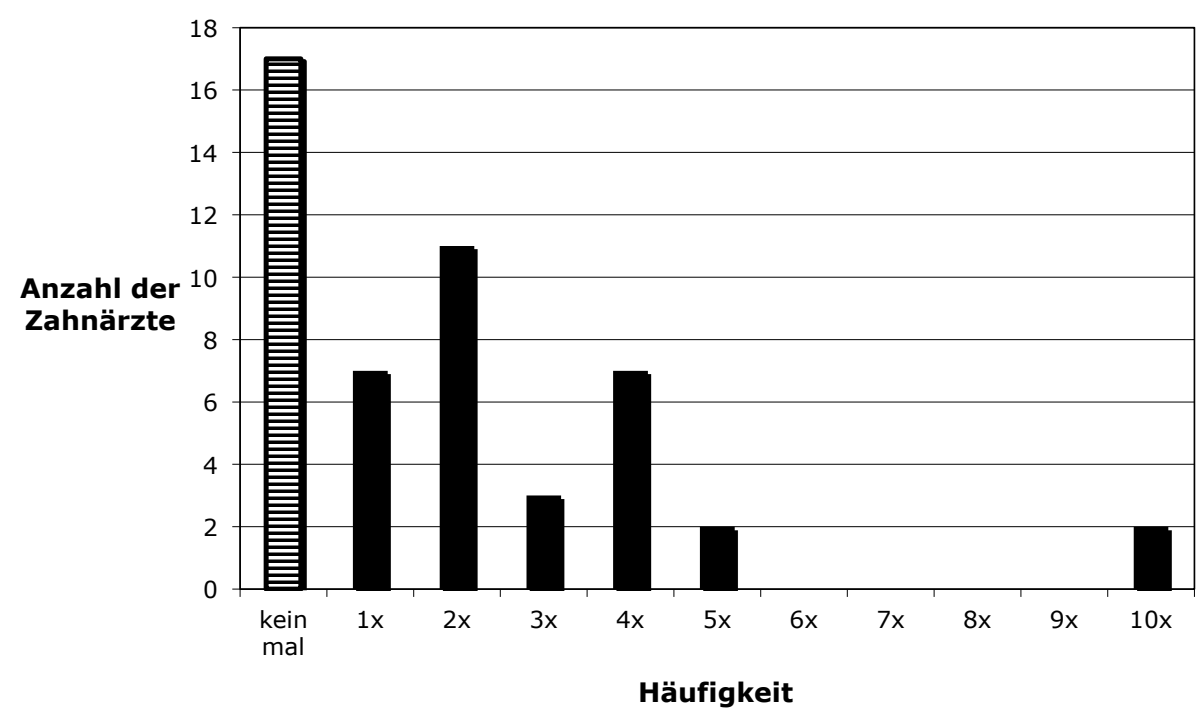

In der Frage Nr. 2 des Fragebogens wurden die Zahnärzte gebeten einzuschätzen, wie viele Patienten sich mit einer bekannten Trigeminusneuralgie im zu Grunde gelegten Zeitraum von 2003 bis 2009 bei ihnen in Behandlung befanden. 48 Zahnärzte beantworteten diese Frage und gaben eine Gesamtzahl von 93 Patienten an. 40 \% der Zahnärzte haben in dieser Zeit keinen Patienten behandelt, der an einer Trigeminusneuralgie litt (Abb. 3.4), etwa genauso viele $(43,7 \%)$ haben bis zu drei TN-Patienten in diesem Zeitraum behandelt. 
Es kann gezeigt werden, dass über die Hälfte der Patienten (52,7 \%) von nur 8 Zahnärzten betreut wurde und dass fast 1/4 (23,7 \%) der Patienten von nur 2 der 48 Zahnärzte behandelt wurde.

Abbildung 3.4: Frage Nr. 2 - beim Zahnarzt in Behandlung mit bekannter TN

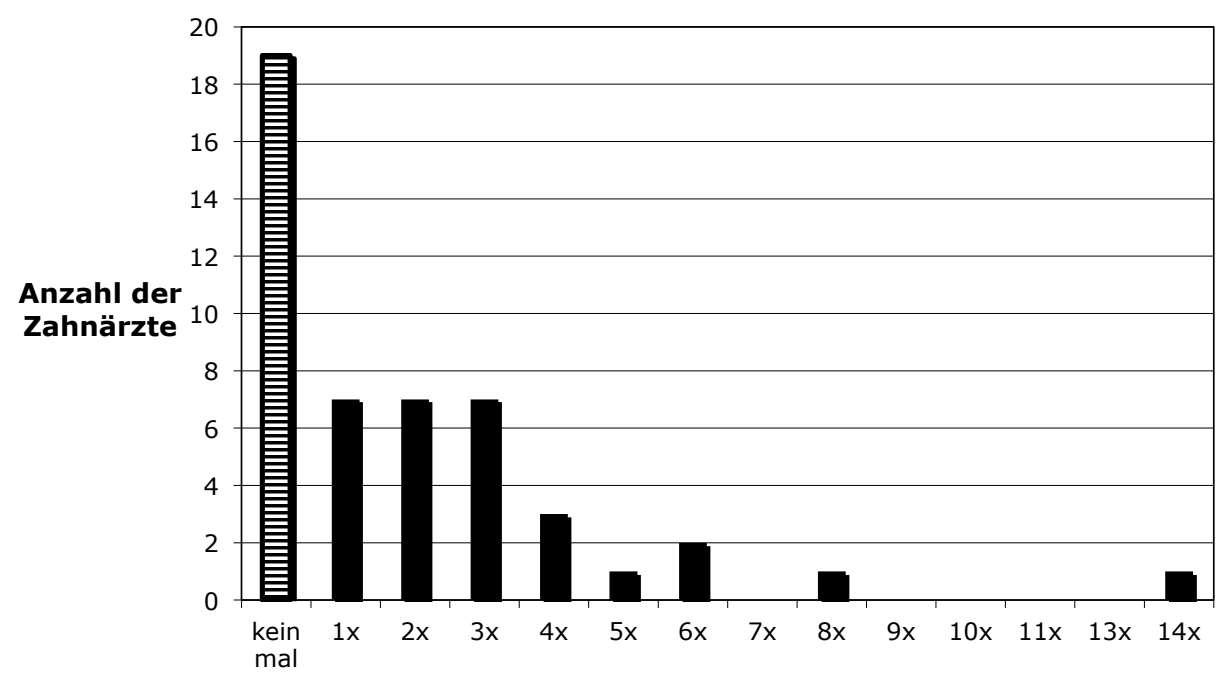

Häufigkeit

Die Fragen Nr. 3 bis Nr. 7 waren geschlossene Fragen, die mit Ja oder Nein beantwortet werden konnten. So sollten die Zahnärzte in der Frage Nr. 3 eine Selbsteinschätzung abgeben, ob sie eine Trigeminusneuralgie erkennen würden. Die Frage wurde von 35 der 47 Zahnärzte mit Ja beantwortet, das entspricht 74,5\%.

Das Medikament Carbamazepin (Frage Nr. 4) war der Hälfte der ingesamt 50 antwortenden Zahnärzte bekannt, genauso wie die Hälfte der Zahnärzte auch Kenntnis über chirurgische Ansätze der Therapie haben (Frage Nr. 5).

Tabelle 3.8: Fragen Nr. 3 bis Nr. 5 des Fragebogens Zahnärzte

\begin{tabular}{|c|c|c||c|c||c|c|}
\hline Nr. & Frage in Kurzform & {$[\mathrm{n}]$} & Ja & $\%$ & Nein & $\%$ \\
\hline \hline 3 & Würden Sie eine TN erkennen? & 47 & 35 & 74,5 & 12 & 25,5 \\
\hline 4 & Kennen Sie Carbamazepin? & 50 & 25 & 50 & 25 & 50 \\
\hline 5 & Kennen Sie auch chirurgische Ansätze? & 50 & 25 & 50 & 25 & 50 \\
\hline
\end{tabular}


Die Frage, ob ein allgemeiner Fortbildungsbedarf zum Thema Trigeminusneuralgie in der Zahnarztpraxis besteht, bejahten 76,6 \% von 47 Zahnärzten (Frage Nr. 6).

In der letzten Frage (Nr. 7) des Fragebogens Zahnärzte, wurden die Zahnärzte gefragt, ob sie gegebenenfalls für ein kurzes Telefonat zum Thema Trigeminusneuralgie oder für Rückfragen zur Verfügung stehen. 55,8 \% der Zahnärzte erklärten sich dazu bereit. 


\section{Diskussion}

Das Ziel der Studie war herauszufinden, ob TN-Patienten bedingt durch ihre Schmerzsymptomatik als erstes eine zahnärztliche Einrichtung aufsuchen und für den Fall, dass dies zutrifft, wie sich der weitere Behandlungsablauf gestaltet.

\subsection{Limitationen}

Bevor mit der Diskussion der gewonnen Erkenntnisse begonnen werden kann, muss auf die größten Limitationen der durchgeführten Studie hingewiesen werden.

Die zugrundeliegenden Informationen wurden in Patienteninterviews gesammelt. Es wurde keine Einsicht in die Karteisysteme der behandelnden Zahnärzte bezüglich damaligen Zahnstatus, Röntgenaufnahmen oder anderweitiger Befunde genommen. Somit kann nicht ausgeschlossen werden, dass individuelle zahnärztliche Behandlungsmaßnahmen klar indiziert und gerechtfertigt waren. Es wurde zwar mit der Patientenauswahl ab 2003 berücksichtigt, dass seit dem möglichen Zahnarztbesuch keine zu große Zeit vergangen ist - da die Erhebung aber auf der Erinnerung der Patienten beruht, besteht die Möglichkeit, dass konkurrierende dentale Pathologien oder prophylaktische Maßnahmen dem Patienten bei der Befragung als solche nicht mehr präsent waren.

Auch konnten viele Patienten Angaben zum genauen zeitlichen Ablauf nur an Hand von Schätzungen vornehmen.

Des Weiteren ist nicht auszuschließen, dass die damalige, neurochirurgische Diagnose TN bei unseren Patienten inkorrekt war. Da jedoch zwei Drittel der aufgrund dieser Diagnose operierten Patienten angaben, beschwerdefrei zu sein, was mit den Ergebnissen in der Literatur bei perkutaner Rhizotomie und MVD vergleichbar ist (Montano et al. 2012), kann die Zahl der Fehldiagnosen als gering eingeschätzt werden.

\subsection{Diskussion der Ergebnisse}

In dieser Studie wurden retrospektive telefonische Interviews mit Patienten geführt, die in der Abteilung Neurochirurgie der UMG aufgrund einer TN operiert wurden. Die Patienten wurden abhängig von der durchgeführten OP-Technik in die OP-Gruppen I 
oder II eingeteilt. Die Analyse der Ergebnisse ergab für die zu untersuchenden Fragen keine signifikanten Unterschiede zwischen den Gruppen, so dass die erwähnte Unterteilung in der Bewertung der Ergebnisse keine Rolle spielt.

In unserer Studie wurden alle Teilnehmer als erstes zur Regelmäßigkeit ihrer Zahnarztbesuche befragt und $80 \%$ gaben an, regelmäßig beim Zahnarzt zu Vorsorgeuntersuchungen zu erscheinen. Als regelmäßig wurde der jährliche Zahnarztbesuch angesehen. Somit konnte ein Eindruck vom Verhältnis der Teilnehmer zum Zahnarzt gewonnen werden und sichergestellt werden, dass nicht ein Großteil der Befragten aufgrund von Angst oder aus anderen Gründen den Zahnarztbesuch per se vermeidet.

\subsubsection{TN-Patienten gehen als erstes zum Zahnarzt}

Mit der zweiten Frage konnten wir herausfinden, dass $82 \%$ der Befragten als erstes mit ihren Beschwerden zum Zahnarzt gingen. Damit konnte die anfangs aufgestellte Hypothese bestätigt werden. Diese Erkenntnis deckt sich mit einer ähnlichen Studie, die in den 80er Jahren in der Schweiz durchgeführt wurde (Garvan und Siegfried 1983). In dieser Studie sind 140 Patienten, welche über einen längeren Zeitraum mit Carbamazepin auf Grund einer angenommenen TN behandelt wurden, zum Behandlungsverlauf seit dem ersten Schmerz befragt worden. Von den 140 Patienten sind $73 \%$ als erstes zum Zahnarzt gegangen. Es muss allerdings in Frage gestellt werden, ob alle Teilnehmer in dieser Studie nach heutigen Erkenntnissen unter einer idiopathischen TN gelitten haben, da als Auswahlkriterien lediglich die regelmäßige Einnahme von Carbamazepin seit 2 Monaten und das Vorhandensein der Schmerzsymptomatik seit 12 Monaten angesetzt wurden. Differentialdiagnostisch kommen dentale Ursachen, die Craniomandibuläre Dysfunktion und die postzosterische Neuralgie in Frage (Baron et al. 2010, Zakrzewska 2002).

In einer Studie von Türp et al. (1998) wurde gezeigt, dass 206 Patienten mit persistierenden Gesichtsschmerzen, die an eine Schmerzklinik mit Universitätsanschluss überwiesen wurden, zuvor durchschnittlich 4.88 Vorbehandler hatten, wovon $70 \%$ aus dem zahnmedizinischen Bereich kamen. Auch hier ist einzuschränken, dass kein Unterschied zwischen Patienten mit TN oder anderen chronischen Gesichtsschmerzen gemacht wurde.

Es konnte mit unserer Studie gezeigt werden, dass der Zahnarzt weiterhin als erster Ansprechpartner bei Patienten mit TN dient, wobei bei einer so seltenen Krankheit eine große Veränderung im Wissensstand der zentraleuropäischen Bevölkerung auch nicht unbedingt zu erwarten war.

Damit wird deutlich, welch wichtige Rolle dem Zahnarzt bei der Früherkennung einer Trigeminusneuralgie zufällt. 


\subsubsection{TN-Patienten werden zahnmedizinisch behandelt}

Alle Patienten, die als Erstdiagnostiker den Zahnarzt angegeben haben, wurden in unserer Studie anschließend zum weiteren Behandlungsablauf befragt. Herauszufinden galt, was Zahnärzte unternehmen, wenn sich Patienten mit Symptomen vorstellen, denen eine TN zugrunde liegt. Es konnte festgestellt werden, dass $60 \%$ der TN-Patienten, die einen Zahnarzt aufsuchten auch eine invasive und meist irreversible Behandlung erfahren haben. Dies deckt sich ebenfalls mit der zuvor erwähnten Studie von Garvan und Siegfried (1983), bei welcher $66 \%$ der Patienten eine Behandlung erfahren haben. Hierbei handelte es sich vor allem um die Extraktion von Zähnen, mit dem Unterschied, dass damals bei 140 Patienten der schweizerischen Studie 680 Zähne gezogen wurden im Vergleich zu 54 Zähne bei 51 Patienten in der vorliegenden Untersuchung. In unserer Studie wurden zusätzlich auch andere Behandlungsformen abgefragt, wobei 13 Wurzelkanalbehandlungen bei 51 Patienten hervorzuheben sind.

Ebenfalls von Siegfried (2000) wurde aufgezeigt, dass in einer späteren Reihenuntersuchung von 1000 Fällen den Patienten insgesamt 380 Zähne gezogen wurden.

Merrill und Graff-Radford (1992) stellten in ihrer Studie fest, dass 61\% ihres Patientenkollektivs mit TN zuvor zahnärztlich behandelt wurden und das an diesen 38 Patienten 24 Wurzelkanalbehandlungen durchgeführt und eine nicht genannte Anzahl von Zähnen extrahiert wurden. Im selben Jahr veröffentlichte Shiotani (1992) seine Studie über die TN und führte aus, dass von 3214 Patienten 34,4 \% zuvor Zähne extrahiert bekommen haben. Rund 12 Jahre später fand Truelove (2004) heraus, dass bei mehr als einem Drittel von 50 Patienten mit der Diagnose neuropathischer Trigeminusschmerz zuvor eine Wurzelbehandlung durchgeführt wurden. Er weist auch auf die Schwierigkeiten hin, eine korrekte Diagnose zu entwickeln im Hinblick auf die signifikanten klinischen Schnittflächen zwischen neuropathischem Trigeminusschmerz, odontogenem Schmerz und Craniomandibulärer Dysfunktion. Es muss erwähnt werden, dass in dieser Studie keine Unterteilung des Patientenkollektivs vorgenommen wird, allerdings sind die detailliert beschriebenen Symptome unter gewissen Umständen einer TN zuzuordnen. Sehr wahrscheinlich wären einige der Patienten, wenn von einem Neurologen oder Neurochirurgen untersucht, TN-spezifisch neurochirurgisch behandelt worden.

Es gilt festzuhalten, dass Patienten nicht nur als erstes einen Zahnarzt aufsuchen, sondern dort auch in vielen Fällen Behandlungen in Form von Extraktionen oder Wurzelkanalbehandlungen erfahren. Nicht außer Acht lassen sollte man dabei, dass Patienten mit einer TN unter einem enorm hohen Leidensdruck stehen. Jeder zweite TN-Patient hat nach eigenen Angaben suizidale Gedanken und jeder vierte einen Suizidversuch hinter sich (Stienen et al. 2010). Oftmals wird der Zahnarzt förmlich um eine Behandlung angefleht (Matwychuk 2004).

Des Weiteren ist die Differentialdiagnostik bei der seltenen TN ein äußerst komple- 
xes Thema, welches noch heute Gegenstand vieler Untersuchungen ist (Baron et al. 2010, Cheng et al. 2011, Jha und Kumar 2012, Zakrzewska 2010 a). Der Diagnostiker muss in einem komplexen anantomischen Gebiet, bei möglichen psychischen Begleiterkrankungen und zentraler Schmerzregulierung eine Fülle von Differentialdiagnosen in Erwägung ziehen.

Im Lichte dessen scheint es nicht verwunderlich, dass in unserer Studie nur bei 2 von 43 befragten Patienten die Verdachtsdiagnose TN vom Zahnarzt gestellt wurden. Die Schwierigkeiten der Diagnosestellung und unnötige Zahnarztbehandlungen waren, laut Mumford (1978), schon zu seiner Zeit allgemein bekannt. Heute finden sich in vielen Studien, auch in zahnmedizinischen Fachzeitschriften (Siegfried 2000, Zakrzewska 2007), zum Thema „Diagnostik der TN“ Hinweise auf die besondere Rolle, die den Zahnärzten zufällt (Spencer et al. 2008).

Trotz alledem fehlt es scheinbar an klaren Leitlinien, an standardisierten Behandlungsprotokollen, die den Zahnärzten durch das Aufstellen der korrekten Verdachtsdiagnose die passende Überweisung ermöglichen. Ansätze und Vorschläge speziell für den Zahnarzt sind in mehreren Studien gemacht worden. So ist allgemeiner Konsens, dass eine ausführliche und gewissenhafte Anamneseerhebung die Grundlage für das Erkennen einer TN ist (Zakrzewska 2010 a). Darauf folgen muss ein ausführlicher extraoraler und dentaler Befund (Clark 2006).

Es soll nicht unerwähnt bleiben, dass Fälle beschrieben wurden, in denen Patienten fälschlicherweise TN-spezifisch behandelt worden sind, obwohl ein atypischer Gesichtsschmerz anderer Genese vorlag. Mumford (1978) beschrieb Fälle von nicht durchgebrochenen Zähnen oder Residualzysten und Roberts el al. (1984) fanden Knochenkavitäten und Fistelgänge bei Patienten, bei denen zuvor eine TN diagnostiziert worden war. Shankland (2001) fand zudem heraus, dass Osteonekrosen im Kieferbereich oftmals TN-ähnliche Symptome hervorrufen. Der Großteil dieser Funde datiert allerdings 15 und mehr Jahre zurück und kann durch die gut abgewägte Nutzung moderner bildgebender Verfahren heutzutage nahezu ausgeschlossen werden (Shintaku et al. 2006).

Sollte vom Zahnarzt keine odontogene Ursache auffindbar sein oder die Beseitigung einer pathologischen Veränderung keine Veränderung der Schmerzsymptomatik hervorrufen, muss an eine neuropathische Ursache gedacht werden. Eine Überweisung in eine neurologische beziehungsweise neurochirurgische Einrichtung oder, wenn vorhanden, in eine Schmerzklinik mit interdisziplinärer Ausrichtung (Ettlin et al. 2007, Koling 1998), muss sich anschließen. 


\subsubsection{TN-Patienten kommen spät in neurologogische oder neurochirurgische Einrichtungen}

In unsere Untersuchung konnte aufgezeigt werden, dass es bei fast der Hälfte $(46,4$ \%) der befragten TN-Patienten mehr als ein Jahr gedauert hat, bis sie in einer neurologischen oder neurochirurgischen Einrichtung vorstellig wurden. Betrachtet man das gesamten Patientenkollektiv sind letztendlich $30 \%$ vom Zahnarzt in eine solche Einrichtung überwiesen worden.

Das Überweisungsverhalten von Zahnärzten war bisher weder im Falle der Verdachtsdiagnose TN noch, mehr allgemein, bei neuralgischen Gesichtsschmerzen Gegenstand von genaueren Untersuchungen. In der Literatur finden sich nur zwei Studien, die dieses Thema aufgreifen. So hat Larner (2011) das Überweisungsverhalten von Mund-, Kiefer-, Gesichtschirurgen (MKG-Chirurgen) zu neurologischen Einrichtungen untersucht und herausgefunden, dass sie nur 12 von 2995 Überweisungen, also weniger als $1 \%$, ausmachen. Hierbei handelt es sich in 5 von 12 Fällen um Patienten mit TN. Ein Studie aus Großbritannien von Pemberton et al. (2001) zeigt, dass nur $5 \%$ der befragten MKG-Chirurgen Patienten mit einer angenommenen TN routinemäßig zum Neurologen oder Neurochirurgen überweisen, $3 \%$ sogar niemals. Die restlichen $92 \%$ führen zumindest manchmal eine solche Überweisung aus.

Es fehlt demnach an größer angelegten, zahnarztspezifischen Untersuchungen bezüglich der Zeit, die vergeht, bis TN-Patienten die richtige Diagnose erhalten. Mann muss sich vor Augen führen, dass eine schnellere Diagnose eine schnellere Schmerzausschaltung und damit eine Verbesserung der Lebensqualität für Patienten mit TN bedeutet. Außerdem führt eine frühe Diagnose wahrscheinlich dazu, dass es zu weniger inadäquaten Therapien kommt und dass die Möglichkeit gegeben ist, durch ein früheres chirurgisches Eingreifen das Resultat der Operation zu verbessern (Barker et al. 1996, Toda 2008, Zakrzewska und Patsalos 2002).

Damit zeigt sich, dass bei der Verbesserung der Situation von Trigeminuspatienten den Zahnärzten eindeutig eine der Schlüsselpositionen in der langen Kette der Ereignisse zukommt.

\subsubsection{Zahnärzte}

50 von 96 angeschriebenen niedergelassenen Zahnärzten im Raum Göttingen haben bei der schriftlichen Umfrage teilgenommen und wurden zum Thema TN befragt.

Es konnte gezeigt werden, dass $74 \%$ der Befragten glauben, eine TN selbstständig erkennen zu können. Betrachtet man eine Studie von Aggarwal et al. (2011), scheint diese Einschätzung durchaus nachvollziehbar. In dieser Studie, in welcher 212 niedergelassene Zahnärzte in Großbritannien zum Thema chronischer orofazialer Gesichtsschmerz 
(COFP) befragt wurden, wurde den Zahnärzten ein ausreichendes Wissen zur richtigen Diagnose dieser Krankheitsgruppe attestiert. Auffällig war, dass die TN, eine Differentialdiagnose des COFP, in dieser Studie von fast $60 \%$ der Befragten fälschlicherweise der Krankheitsgruppe COFP zugeordnet wurden. Die Autoren weisen auf mögliche Wissenslücken hin und empfehlen eine bessere Integration des COFP in den Lehrplan der Universitäten. Allerdings herrscht, anders als von den Autoren dargestellt, in der Wissenschaft noch keine Einigkeit über die genaue Definition von COFP (Benoliel et al. 2010).

In unserer Studie wurde speziell Wissen zum Thema TN erfragt und es zeigte sich, dass nur jeweils der Hälfte der Zahnärzte das Medikament Carbamazepin sowie chirurgische Therapieansätze bekannt waren. Dies weist auf gewisse Wissenslücken in diesem speziellen Themenkomplex hin. In der Literatur lassen sich keine Studien finden, die sich näher mit dem Wissensstand der Zahnärzte zum Thema TN befassen.

Zieht man hinzu, dass 76,6 \% der von uns befragten Zahnärzte zum Thema „TN in der Zahnarztpraxis" einen erhöhten Fortbildungsbedarf sehen, scheint eine weitere Erklärung für die Ergebnisse der zuvor abgehandelten Patientenbefragung gefunden. Die Zahnärzte unterschätzen möglicherweise die Komplexität der Diagnosefindung TN, vermuten aber zu Recht, dass noch Fortbildungsbedarf besteht.

Leider erfahren TN-Patienten auch heute noch unnötige, irreversible zahnärztliche Behandlungen und werden später als nötig in neurologischen bzw. neurochirurgischen Einrichtungen vorstellig. Die Zahnärzte vertrauen zwar in ihr Wissen bei der Erkennung einer TN, sehen aber selbst des Bedürfnis für mehr Fortbildung. Eine Vertiefung des Wissens über die TN mittels Fortbildungsveranstaltungen für Zahnärzte könnte ein effektives Mittel zur Vebesserung der Situation der TN-Patienten sein.

\subsubsection{Schlussfolgerung}

Die Rolle des Zahnarztes in der komplexen Differentialdiagnostik von chronischen Gesichtsschmerzen und das Vorhandensein von nicht zielführenden zahnärztlichen Behandlungen bei TN-Patienten sind Variablen, die vielen Autoren zum Thema TN bekannt zu sein scheinen, und doch ist dies bisher noch allzu wenig genauer untersucht worden. Es fehlen randomisierte kontrollierte Studien zu den Themen therapeutische Intervention von Zahnärzten bei TN-Fällen, dem Überweisungsverhalten von Zahnärzten und dem TN-spezifischen Wissensstand von Zahnärzten. Es ist zu vermuten, dass sich durch eine bessere universitäre Ausbildung und spätere Fort- und Weiterbildung zum Thema TN die Leidenszeiten von TN-Patienten verkürzen und unnötige zahnärztliche Eingriffe vermeiden lassen. 
Die enge Zusammenarbeit von Neurologen, Neurochirurgen und Zahnärzten in der Einschätzung herausfordernder Fälle scheint eine wichtige Voraussetzung zu sein. So können zum Beispiel interdisziplinäre Schmerzzentren ein geeignetes Instrument sein, um an einem Ort mit hoher Spezialisierung schneller zur korrekten Diagnose zu gelangen. 


\section{Zusammenfassung}

Personen mit einer TN leiden unter einseitigen, blitzartig einschießenden, extrem heftigen, elektrisierenden und stechenden Schmerzen im Versorgungsgebiet eines oder mehrerer Äste des N. trigeminus, welche durch Sprechen, Rasieren oder Schlucken, aber auch durch Kauen, Zähneputzen und Berührungen in der perioralen Region ausgelöst werden können.

Es handelt sich bei der TN um eine seltene Krankheit, deren Ursache noch nicht abschließend geklärt ist, sich aber zufriedenstellend medikamentös durch die Gabe von Antikonvulsiva wie Carbamazepin oder Oxcarbazepin kontrollieren lässt. Bei unzureichender Schmerzkontrolle oder bei zu starken Nebenwirkungen stehen mehrere chirurgische Techniken zur Auswahl, wovon die MVD die längsten postoperativen schmerzfreien Intervalle verspricht.

Der Neurologe oder der Neurochirurg sollte folglich der erste Ansprechpartner sein, wenn der Verdacht einer TN besteht.

Führt man sich jedoch die Symptomatik der TN in Kombination mit ihrem geringen Bekanntheitsgrad vor Augen, lässt sich vermuten, dass die TN von Patienten und deren Verwandten nicht als solche erkannt wird und aufgrund des betroffenen Gebietes ein Zahnarzt aufgesucht wird.

Sollte sich diese zu untersuchende Hypothese bewahrheiten und sich der Zahnarzt als initialer Arztkontakt bei TN-Patienten herausstellen, ist die Versorgung dieser Erkrankung im weiteren Verlauf entscheidend abhängig vom Verhalten des behandelnden Zahnarztes. Um einen Eindruck vom Behandlungsverlauf zu bekommen gilt also zusätzlich herauszufinden, wie mit TN-Patienten beim Zahnarzt verfahren wird und wie Zahnärzte selbst die Situation und sich selbst einschätzen.

Im Rahmen dieser retrospektiven Studie sind 57 Personen mit der Diagnose TN, die in den Jahren 2003-2009 in der UMG operiert wurden, anhand eines standardisierten Fragebogens telefonisch interviewt worden. Es wurde die Regelmäßigkeit ihrer vorherigen Zahnarztbesuche abgefragt und ihre individuelle TN-Krankengeschichte mit speziellem Fokus auf mögliche zahnärztliche Konsultationen und Behandlungen untersucht. Des Weiteren wurden 51 im Raum Göttingen niedergelassene Zahnärzte zum Thema TN befragt und um ihre Einschätzung gebeten.

Es konnte herausgefunden werden, dass $82 \%$ der befragten TN-Patienten als erstes 
einen Zahnarzt aufsuchten. 60 \% der Patienten berichteten, zusätzlich eine Behandlung in Form von invasiven Maßnahmen erfahren zu haben. Zu den Maßnahmen zählten vor allem Extraktionen (54 Zähne bei 51 Patienten) und Wurzelkanalbehandlungen (13 bei 51 Patienten).

Außerdem konnte festgestellt werden, dass nur 2 Patienten erinnerlich war, dass der Zahnarzt die Verdachtsdiagnose TN gestellt hat und dass fast die Hälfte der Befragten erst nach einem Jahr oder länger eine neurologische oder neurochirurgische Einrichtung aufgesucht haben.

Bei den Zahnärzten war auffällig, dass $74 \%$ sich selbst so einschätzen, dass sie eine TN erkennen würden. Das Medikament Carbamazepin und chirurgische Verfahren in der TN-Therapie kannten hingegen nur jeweils die Hälfte der Befragten. Besonders festzuhalten ist, dass $77 \%$ der Zahnärzte selbst einen weiteren Fortbildungsbedarf zum Thema „TN in der Zahnarztpraxis“ sehen.

Einschränkend ist zu unseren Ergebnissen zu sagen, dass die Datenerhebung mittels telefonischer Interviews durchgeführt wurde und keine Kontrolle der zahnärztlichen Befunde und Röntgenbilder stattgefunden hat. Es ist somit nicht auszuschließen, dass etwaige zahnärztliche Behandlungen klar indiziert waren, sei es aufgrund einer konkurrierenden dentalen Pathologie oder aus prophylaktischen Gründen, die dem Patienten so nicht mehr erinnerlich waren.

Des Weiteren besteht die Möglichkeit, dass die neurochirurgische Diagnose der befragten Patienten ursprünglich falsch gewesen war. Die große Beschwerdefreiheit der Patienten nach der chirurgischen Therapie lässt die Zahl solcher Fälle jedoch gering erscheinen.

Es bleibt festzuhalten, dass trotz zunehmendem Kenntnisgewinn beim Krankheitsbild TN auch heute noch TN-Patienten irreversible zahnärztliche Behandlungen erfahren und später als nötig in neurologischen Einrichtungen vorstellig werden. Da Zahnärzte diese Patienten in der Regel als erstes sehen, kann und muss an dieser Stelle angesetzt werden.

Unsere Untersuchungen lassen Wissenslücken der Zahnärzte beim Thema TN erahnen, welche weiterführend in größer angelegten Studien validiert werden müssen. Außerdem verdient das Überweisungsverhalten von Zahnärzten und anderen Spezialisten im Verdachtsfall TN eine genauere Betrachtung, um den stark leidenden Patienten mit TN eine möglichst rasche, adäquate Therapie zukommen zu lassen. 


\section{Anhang}

\subsection{Abkürzungsverzeichnis}

${ }^{\circ} \mathrm{C} \quad$ Grad Celsius

COFP Chronical orofacial pain

Gy Gray

IHS International Headache Society

ICHD-2 International Classification of Headache Disorders, Second Edition

m Median

MKG Mund, Kiefer, Gesicht

MRT Magnetresonanztomographie

MVD Mikrovaskuläre Dekompression

N. Nervus

[n] Anzahl

OP Operation

SUNA Short unilateral neuralgiform pain with autonomic symptoms

SUNCT Short unilateral neuralgiform pain with conjunctival redness and tearing

TN Trigeminusneuralgie

UMG Universitätsmedizin Göttingen

WBH Wurzelkanalbehandlung

WHO World Health Organization

ZA Zahnarzt 


\subsection{Tabellenverzeichnis}

3.1 Ja/Nein-Fragen Fragebogen Patienten (gesamt) 19

3.2 Überweisungen der Zahnärzte 21

3.3 Intervall zwischen Auftreten erster Symptome und Zahnarztbesuch (1. $\rightarrow$ 2.) 22

3.4 Intervall zwischen Zahnarztbesuch und neurolog./neurochirurg.

Begutachtung $(2 . \rightarrow 3$.)

3.5 Intervall zwischen Auftreten erster Symptome und neurolog./neurochirurg.

Begutachtung (1. $\rightarrow$ 3.) 22

3.6 Ja/Nein- Fragen Fragebogen Patienten (OP-Gruppe I) 23

3.7 Ja/Nein-Fragen Fragebogen Patienten (OP-Gruppe II) 24

3.8 Fragen Nr. 3 bis Nr.5 des Fragebogens Zahnärzte 26

\subsection{Abbildungsverzeichnis}

1.1 Intraoperative Darstellung eines Gefäß-Nervenkontaktes am N. trigeminus 3

$\begin{array}{ll}1.2 \text { MRT bei Gefäßkompression des N. trigeminus rechts } & 7\end{array}$

1.3 Lage der Kanüle bei Gangliolyse (von lateral) 10

1.4 Lage der Kanüle bei Gangliolyse (Schrägaufnahme) 11

3.1 Therapiemaßnahmen der Zahnärzte 20

3.2 Verteilung der durchgeführten Extraktionen 20

3.3 Frage Nr. 1 - Häufigkeit der Verdachtsdiagnose TN pro Zahnarzt im Zeitraum 2003-2009 25

3.4 Frage Nr. 2 - beim Zahnarzt in Behandlung mit bekannter TN (2003-2009) 26

Die Abbildungen 1.1 - 1.4 wurden verwendet mit freundlicher Genehmigung von

Dr. Kajetan von Eckardstein, Zentrum neurologische Medizin, Universitätsmedizin Göttingen. 


\subsection{Anschreiben Patienten}

Sehr verehrte Patientin, sehr geehrter Patient,

Im Rahmen einer Doktorarbeit in der Klinik für Neurochirurgie der Universitätsmedizin Göttingen benötigen wir Ihre Hilfe und Zusammenarbeit. Wir möchten herausfinden, wie oft Patienten mit einer Trigeminusneuralgie zuvor aufgrund ihrer Gesichtsschmerzen eine Zahnärztin/einen Zahnarzt aufgesucht haben, welche Maßnahmen ergriffen wurden und inwieweit die zahnärztliche Behandlung erfolgreich war. Ihren Namen und Ihre Adresse haben wir aus unserem Patientenarchiv entnehmen können, da Sie bei uns in der Vergangenheit an einer Trigeminusneuralgie behandelt wurden.

Zu diesem Zweck haben wir einen kurzen Fragebogen entworfen, den der Doktorand Herr Moritz Keil gerne mit Ihnen telefonisch durchgehen würde. Es wird sich dabei um ein Gespräch von maximal zehn Minuten handeln. Auch wenn Sie sich nicht in zahnärztlicher Behandlung befanden, ist Ihre Teilnahme für uns wichtig. Sämtliche Informationen werden selbstverständlich vertraulich behandelt und ausschließlich in anonymisierter Form verwendet und ausgewertet. Sie könnten mit Ihrer Teilnahme an der Befragung einen Beitrag leisten, um den Umgang von Zahnärzten mit Menschen, die unter einer Trigeminusneuralgie leiden, zu verbessern.

Das Forschungsvorhaben ist von der Ethik-Kommission der Universität Göttingen überprüft und genehmigt worden.

Für den Fall, dass Sie dabei helfen möchten, würden wir Sie bitten, die umseitige Einwilligung zu lesen, zu unterschreiben und im bereits frankierten Rückumschlag zurückzusenden. Sollte Ihnen ein bestimmter Tag oder eine Uhrzeit besonders passen, können Sie dies gerne vermerken.

Herr Keil freut sich sehr auf ein Gespräch mit Ihnen.

Mit freundlichen Grüßen

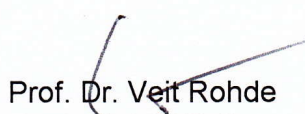
Direktor der Klinik

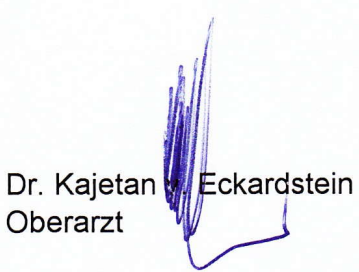

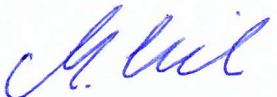

cand. med. dent. Moritz Keil Doktorand 


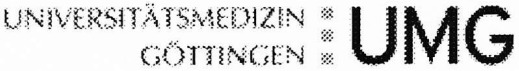

\section{Einwilligung}

Hiermit bestätige ich, dass ich an der umseitig beschriebenen Studie („Die Trigeminusneuralgie als Differentialdiagnose zum odontogenem Schmerz aus zahnmedizinischer Sicht") teilnehmen möchte und damit einverstanden bin, dass Herr Moritz Keil im Rahmen seiner Doktorarbeit telefonischen Kontakt zu mir aufnimmt.

Ort, Datum, Unterschrift

Am besten erreichbar bin ich an folgenden Tagen bzw. zu dieser Uhrzeit:

Ich möchte gerne unter der folgenden Telefonnummer kontaktiert werden:

Herzlichen Dank für Ihre Mithilfe! 


\subsection{Fragebogen Patienten}

\section{$\underline{\text { Patientenfragebogen }}$}

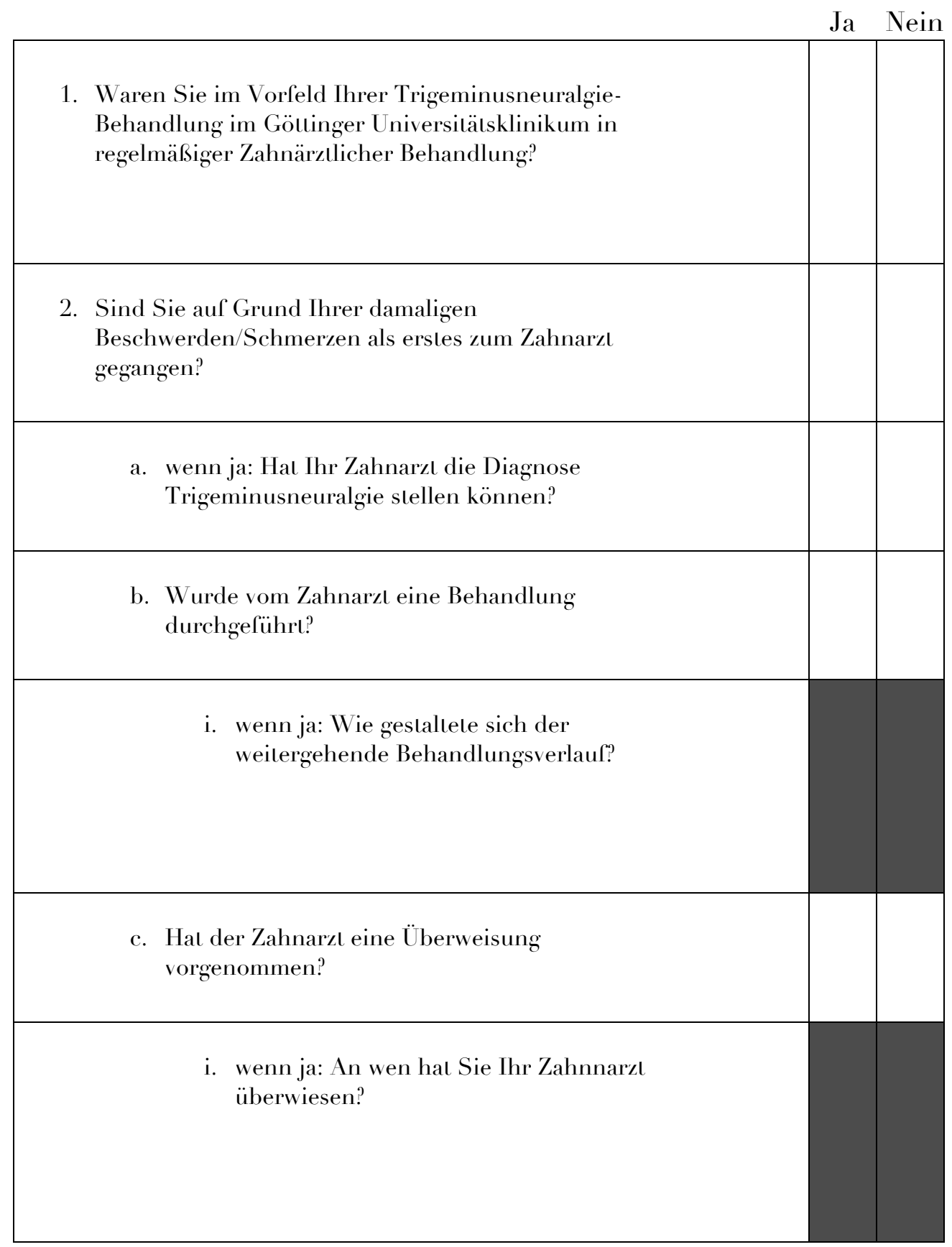




\begin{tabular}{|l|l|}
\hline a. Können sie sagen, wie viel Zeit zwischen (1.) \\
den ersten Beschwerden, (2.) dem \\
Zahnarztbesuch und (3.) einer \\
neurochirurgischen Begutachtung vergangen \\
ist? \\
1. \\
2. \\
3. & \\
\hline 3. Haben Sie momentan Beschwerden? & \\
& \\
\end{tabular}




\subsection{Anschreiben Zahnärzte}

Sehr verehrte Frau Kollegin, sehr geehrter Herr Kollege,

im Rahmen einer in der Neurochirurgischen Klinik der Universitätsmedizin an Herrn Keil, Student der Zahnmedizin, vergebenen Dissertation „Die Trigeminusneuralgie als Differentialdiagnose zum odontogenen Schmerz" benötigen wir Ihre Hilfe und Zusammenarbeit. Ziel ist es herauszufinden, in wie vielen Fällen Patienten mit einer Trigeminusneuralgie initial eine Zahnärztin/einen Zahnarzt konsultieren, wie diese/dieser dem Patienten helfen kann und ob eventuell ein Fortbildungsbedarf besteht.

Aus diesem Grund haben wir umseitig einen kurzen Fragebogen entworfen, den wir Sie bitten würden, ausgefüllt in dem bereits frankierten Umschlag zurücksenden. Wir garantieren für eine diskrete Handhabung und Anonymisierung Ihrer Daten. Eine anonymisierte Rücksendung ist selbstverständlich ebenfalls möglich.

Das Forschungsvorhaben ist von der Ethik-Kommission der Universität Göttingen überprüft und genehmigt worden.

Wir würden uns sehr freuen, eine Antwort von Ihnen zu bekommen. Für etwaige Fragen stehen wir Ihnen gerne zur Verfügung.

Mit freundlichen, kollegialen Grüßen

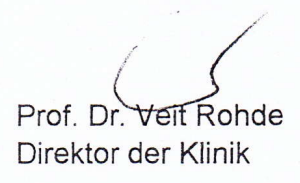

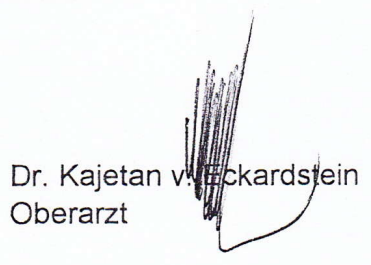

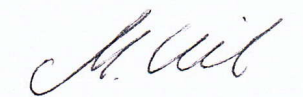

cand. med. dent. Moritz Keil Doktorand 


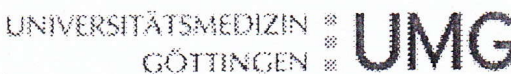

\section{Fragebogen}

1. Wie viele Male in den letzten sechs Jahren konnte von Ihnen schätzungsweise die Verdachtsdiagnose „Trigeminusneuralgie" gestellt werden?

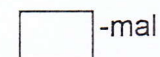

2. Wie viele Patienten mit einer bekannten Trigeminusneuralgie haben Sie ca. in den letzen sechs Jahren behandelt?

Patienten

3. Wie schätzen Sie sich selbst ein: Würden Sie eine Trigeminusneuralgie erkennen? o Ja o Nein

4. Ist Ihnen das Medikament Carbamazepin bekannt?

o Ja o Nein

5. Sind Ihnen neben medikamentösen auch chirurgische Ansätze der Therapie bekannt?

o Ja o Nein

6. Denken Sie, dass ein allgemeiner Fortbildungsbedarf zum Thema „Trigeminusneuralgie in der Zahnarztpraxis" besteht?

o Ja o Nein

7. Stehen Sie ggf. für ein kurzes Telefonat zum Thema Trigeminusneuralgie bzw. für Rückfragen zur Verfügung? (Im positiven Falle dürfen wir dann um Ihren Praxisstempel bitten.)

o Ja o Nein

Herzlichen Dank für Ihre Mithilfe! 


\section{Literaturverzeichnis}

Aggarwal V R, Joughin A, Zakrzewska J M, Crawford F J, Tickle M (2011): Dentists' and specialists' knowledge of chronic orofacial pain: results from a continuing professional development survey. Prim Dent Care 18: 41-4

Ali F M, Prasant M, Pai D, Aher V A, Kar S, Safiya T (2012): Peripheral neurectomies: A treatment option for trigeminal neuralgia in rural practice. J Neurosci Rural Pract 3: $152-7$

Ameli N O (1965): Avicenna and trigeminal neuralgia. J Neurol Sci 2: 105-7

Baad-Hansen L (2008): Atypical odontalgia - pathophysiology and clinical management. J Oral Rehabil 35: 1-11

Backonja M (2003): Anticonvulsants for the treatment of neuropathic pain syndromes. Curr Pain Headache Rep 7: 39-42

Barker F G, Jannetta P J, Bissonette D J, Larkins M V, Jho H D (1996): The longterm outcome of microvascular decompression for trigeminal neuralgia. N Engl J Med 334: $1077-83$

Barker F G, Jannetta P J, Bissonette D J, Jho H D (1997): Trigeminal numbness and tic relief after microvascular decompression for typical trigeminal neuralgia. Neurosurgery 40: $39-45$

Baron R, Binder A and Wasner G (2010): Neuropathic pain: diagnosis, pathophysiological mechanisms, and treatment. Lancet Neurol 9: 807-19

Benoliel R, Eliav E and Sharav Y (2010): Classification of chronic orofacial pain: applicability of chronic headache criteria. Oral Surg Oral Med Oral Pathol Oral Radiol Endod 110: 729-37

Bergenheim A T, Hariz M I, Laitinen L V, Olivecrona M, Rabow L (1991): Relation between sensory disturbance and outcome after retrogasserian glycerol rhizotomy. Acta Neurochir (Wien) 111: 114-8

Bergenheim A T, Asplund P, Linderoth B (2012): Percutaneous Retrogasserian Balloon Compression for Trigeminal Neuralgia: Review of Critical Technical Details and Outcomes. (World Neurosurg, im Druck) 
Benoliel R, Eliav E, Sharav Y (2010): Classification of chronic orofacial pain: applicability of chronic headache criteria. Oral Surg Oral Med Oral Pathol Oral Radiol Endod 110: 729-37

Broggi G, Ferroli P, Franzini A, Servello D, Dones I (2000): Microvascular decompression for trigeminal neuralgia: comments on a series of 250 cases, including 10 patients with multiple sclerosis. J Neurol Neurosurg Psychiatry 68: 59-64

Canavero S and Bonicalzi V (1997): Lamotrigine control of trigeminal neuralgia: an expanded study. J Neurol 244: 527

Chemaly D, Lefrançois A, Pérusse R (2000): Oral and maxillofacial manifestations of multiple sclerosis. J Can Dent Assoc 66: 600-5

Cheng A, Kunchur R, Goss A N (2011): Management challenges in psychiatric patients with severe mandibular pain: a case report. Aust Dent J 56: 82-4

Cheshire W P (2001): Fosphenytoin: an intravenous option for the management of acute trigeminal neuralgia crisis. J Pain Symptom Manage 21: 506-10

Clark G T (2006): Persistent orodental pain, atypical odontalgia, and phantom tooth pain: when are they neuropathic disorders? J Calif Dent Assoc 34: 599-609

Cohen A S, Matharu M S, Goadsby P J (2006): Short-lasting unilateral neuralgiform headache attacks with conjunctival injection and tearing (SUNCT) or cranial autonomic features (SUNA)-a prospective clinical study of SUNCT and SUNA. Brain 129: $2746-60$

Cohen J (2005): Role of the neurologist in the evaluation and treatment of patients with trigeminal neuralgia. Neurosurg Focus 18: E2

Cole C D, Liu J K, Apfelbaum R I (2005): Historical perspectives on the diagnosis and treatment of trigeminal neuralgia. Neurosurg Focus 18: E4

Cruccu G, Gronseth G, Alksne J, Argoff C, Brainin M, Burchiel K, Nurmikko T, Zakrzewska J M, American Academy of Neurology Society and European Federation of Neurological Society (2008): AAN-EFNS guidelines on trigeminal neuralgia management. Eur J Neurol 15: 1013-28

Clark G T (2006): Persistent orodental pain, atypical odontalgia, and phantom tooth pain: when are they neuropathic disorders? J Calif Dent Assoc 34: 599-609

Dandy W E (1932): The treatment of trigeminal neuralgia by the cerebellar route. Ann Surg 96: 787-95 
Degn J and Brennum J (2010): Surgical treatment of trigeminal neuralgia. Results from the use of glycerol injection, microvascular decompression, and rhizotomia. Acta Neurochir (Wien), im Druck

Eller J L, Raslan A M, Burchiel K J (2005): Trigeminal neuralgia: definition and classification. Neurosurg Focus 18: E3

Ettlin D, Galli U, Palla S (2007): The Interdiscipilinary Orofacial Pain Consulting Service at the Center for Dental and Oral Medicine and Cranio-Maxillofacial Surgery, University of Zurich, Switzerland. Schweiz Monatsschr Zahnmed 117: 393-408

Fothergill J (1773): Of a painful affection of the face. Med Obs Inq 5: 129-42

Fromm G H, Terrence C F, Chattha A S (1984): Baclofen in the treatment of trigeminal neuralgia: double-blind study and long-term follow-up. Ann Neurol 15: 240-4

Gardner W J (1962): Concerning the mechanism of trigeminal neuralgia and hemifacial spasm. J Neurosurg 19: 947-58

Gardner W J, Miklos M V (1959): Response of trigeminal neuralgia to decompression of sensory root; discussion of cause of trigeminal neuralgia. J Am Med Assoc 170: 1773-6

Garvan N J, Siegfried J (1983): Trigeminal neuralgia-earlier referral for surgery. Postgrad Med J 59: 435-7

Goru S J, Pemberton M N (2009): Trigeminal neuralgia: the role of magnetic resonance imaging. Br J Oral Maxillofac Surg 47: 228-9

Gronseth G, Cruccu G, Alksne J, Argoff C, Brainin M, Burchiel K, Nurmikko T, Zakrzewska J M (2008): Practice parameter: the diagnostic evaluation and treatment of trigeminal neuralgia (an evidence-based review): report of the Quality Standards Subcommittee of the American Academy of Neurology and the European Federation of Neurological Societies. Neurology 71: 1183-90

Harris W (1951): A history of the treatment of trigeminal neuralgia. Postgrad Med J 27: $18-21$

Horowitz M, Horowitz M, Ochs M, Carrau R, Kassam A (2004): Trigeminal neuralgia and glossopharyngeal neuralgia: two orofacial pain syndromes encountered by dentists. J Am Dent Assoc 135: 1427-33; quiz 1468

IASP (1986): Classification of chronic pain. Descriptions of chronic pain syndromes and definitions of pain terms. Prepared by the International Association for the Study of Pain, Subcommittee on Taxonomy. Pain Suppl 3: S1-226

Ibrahim S (2012): Trigeminal neuralgia: diagnostic criteria, clinical aspects and treatment outcomes. A retrospective study. (Gerodontology, im Druck) 
Israel H A, Scrivani S J (2000): The interdisciplinary approach to oral, facial and head pain. J Am Dent Assoc 131: 919-26

Jannetta P J (1967): Arterial compression of the trigeminal nerve at the pons in patients with trigeminal neuralgia. J Neurosurg 26: 159-162

Jannetta P J (1980): Neurovascular compression in cranial nerve and systemic disease. Ann Surg 192: 518-25

Jha A K, Kumar N G (2012): Accessory mental nerve: case report, review and its role in trigeminal neuralgia. Surg Radiol Anat 34: 469-73

Kalkanis S N, Eskandar E N, Carter B S, Barker F G (2003): Microvascular decompression surgery in the United States, 1996 to 2000: mortality rates, morbidity rates, and the effects of hospital and surgeon volumes. Neurosurgery 52: 1251-61; discussion $1261-2$

Katusic S, Beard C M, Bergstralh E, Kurland L T (1990): Incidence and clinical features of trigeminal neuralgia, Rochester, Minnesota, 1945-1984. Ann Neurol 27: 89-95

Kawaura K, Miki R, Urashima Y, Honda S, Shehata A M, Soeda F, Shirasaki T, Takahama K (2011): Tipepidine enhances the antinociceptive-like action of carbamazepine in the acetic acid writhing test. Eur J Pharmacol 651: 106-8

Keane J R (1990): Neurectasy: the short history of therapeutic nerve stretching and suspension. Neurology 40: 829-31

Köling A (1998): Neurologist, otolaryngologist...? Which specialist should treat facial pain? Lakartidningen 95: 2320-5

Kondziolka D, Zorro O, Lobato-Polo J, Kano H, Flannery T J, Flickinger J C, Lunsford L D (2010): Gamma Knife stereotactic radiosurgery for idiopathic trigeminal neuralgia. J Neurosurg 112: 758-65

Koopman J S H A, Dieleman J P, Huygen F J, Mos M, Martin C G M, Sturkenboom M C J M (2009): Incidence of facial pain in the general population. Pain 147: 122-7.

Krafft R M (2008): Trigeminal neuralgia. Am Fam Physician 77: 1291-6

Krause B (2007): The revision process of medical classifications in Germany. Bundesgesundheitsblatt Gesundheitsforschung Gesundheitsschutz 50: 1055-60

Larner A J (2011): Referrals to the neurology clinic from oral and maxillofacial surgery clinics. Br J Oral Maxillofac Surg 49: 576-7

Lemos L, Alegria C, Oliveira J, Machado A, Oliveira P, Almeida A (2011): Pharmacological versus microvascular decompression approaches for the treatment of trigeminal neuralgia: clinical outcomes and direct costs. J Pain Res 4: 233-44 
Lopez B C, Hamlyn P J, Zakrzewska J M (2004): Systematic review of ablative neurosurgical techniques for the treatment of trigeminal neuralgia. Neurosurgery 54: 973-82; discussion 982-3

Love S, Coakham H B (2001): Trigeminal neuralgia: pathology and pathogenesis. Brain 124: $2347-60$

Matwychuk M J (2004): Diagnostic challenges of neuropathic tooth pain. J Can Dent Assoc 70: 542-6.

McLaughlin M R, Jannetta P J, Clyde B L, Subach B R, Comey C H, Resnick D K (1999): Microvascular decompression of cranial nerves: lessons learned after 4400 operations. J Neurosurg 90: 1-8

Merrill R L, Graff-Radford S B (1992): Trigeminal neuralgia: how to rule out the wrong treatment. J Am Dent Assoc 123: 63-8

Montano N, Papacci F, Cioni B, Di Bonaventura R, Meglio M (2012): What is the best treatment of drug-resistant trigeminal neuralgia in patients affected by multiple sclerosis? A literature analysis of surgical procedures. (Clin Neurol Neurosurg, im Druck) Mumford J M (1978): Role of the dentist in trigeminal neuralgia. Pain ㅁ: 83-92

Nurmikko T J (2009): Pathophysiology of MS-related trigeminal neuralgia. Pain 143: $165-6$

Nurmikko T J, Eldridge P R (2001): Trigeminal neuralgia-pathophysiology, diagnosis and current treatment. Br J Anaesth 87: 117-32

Olesen J (2006): International Classification of Headache Disorders, Second Edition (ICHD-2): current status and future revisions. Cephalalgia 26: 1409-10

International Headache Society H C S (2004): The International Classification of Headache Disorders: 2nd edition. Cephalalgia 24 Suppl 1: 9-160

Pagni C A, Fariselli L, Zeme S (2008): Trigeminal neuralgia. Non-invasive techniques versus microvascular decompression. It is really available any further improvement? Acta Neurochir Suppl 101: 27-33

Pemberton M N, Dewi P S, Hindle I, Thornhill M H (2001): Investigation and medical management of trigeminal neuralgia by consultant oral and maxillofacial surgeons in the British Isles. Br J Oral Maxillofac Surg 39: 114-9

Roberts A M, Person P, Chandran N B, Hori J M (1984): Further observations on dental parameters of trigeminal and atypical facial neuralgias. Oral Surg Oral Med Oral Pathol 58: 121-9 
Rose F C (1999): Trigeminal neuralgia. Arch Neurol 56: 1163-4

Prasad S, Galetta S (2009): Trigeminal neuralgia: historical notes and current concepts. Neurologist 15: 87-94

Sarlani E, Grace E G, Balciunas B A, Schwartz A H (2005): Trigeminal neuralgia in a patient with multiple sclerosis and chronic inflammatory demyelinating polyneuropathy. J Am Dent Assoc 136: 469-76

Sarsam Z, Garcia-Fiñana M, Nurmikko T J, Varma T R K, Eldridge P (2010): The longterm outcome of microvascular decompression for trigeminal neuralgia. Br J Neurosurg 24: $18-25$

Shankland W E (2001): Differential diagnosis of two disorders that produce common orofacial pain symptoms. Gen Dent 49: 150-5

Shintaku W, Enciso R, Broussard J, Clark G T (2006): Diagnostic imaging for chronic orofacial pain, maxillofacial osseous and soft tissue pathology and temporomandibular disorders. J Calif Dent Assoc 34: 633-44

Shiotani M (1992): Trigeminal neuralgia. Pain 13: 867-874

Siegfried J (2000): Die Trigeminusneuralgie. Schweiz Monatsschr Zahnmed 110: 10791081

Siniscalchi A, Gallelli L, Avenoso T, Squillace A, De Sarro G (2011): Effects of carbamazepine/oxycodone coadministration in the treatment of trigeminal neuralgia. Ann Pharmacother 45: e33

Sommer C (2002): Pharmacotherapy of orofacial pain. Schmerz 16: 381-8

Spencer C J, Neubert J K, Gremillion H, Zakrzewska J M, Ohrbach R (2008): Toothache or trigeminal neuralgia: treatment dilemmas. J Pain 9: 767-70

Stienen M N, Cadosch D, Seule M A, Fournier J Y, Hildebrandt G, Gautschi O P (2010): Trigeminal neuralgia - pathophysiology, clinical aspects and treatment. Praxis (Bern) 99: 29-43

Sweet W H, Wepsic J G (1974): Controlled thermocoagulation of trigeminal ganglion and rootlets for differential destruction of pain fibers. 1. Trigeminal neuralgia. J Neurosurg 40: 143-56.

Taylor J C, Brauer S, Espir M L (1981): Long-term treatment of trigeminal neuralgia with carbamazepine. Postgrad Med J 57: 16-8

Toda K (2008): Operative treatment of trigeminal neuralgia: review of current techniques. Oral Surg Oral Med Oral Pathol Oral Radiol Endod 106: 788-805, 805.e1-6 
Tronnier V M, Rasche D, Hamer J, Kienle A L, Kunze S (2001): Treatment of idiopathic trigeminal neuralgia: comparison of long-term outcome after radiofrequency rhizotomy and microvascular decompression. Neurosurgery 48: 1261-7; discussion 1267-8

Truelove E (2004): Management issues of neuropathic trigeminal pain from a dental perspective. J Orofac Pain 18: 374-80

Türp J C (2005): Atypical odontalgia. Schweiz Monatsschr Zahnmed 115: 1006-11

Türp J C, Kowalski C J, Stohler C S (1998): Treatment-seeking patterns of facial pain patients: many possibilities, limited satisfaction. J Orofac Pain 12: 61-6

Wilkins R H, Brody I A (1969): Bell's palsy and Bell's phenomenon. Arch Neurol 21: $661-2$

Zacest A C, Magill S T, Miller J, Burchiel K J (2010): Preoperative magnetic resonance imaging in Type 2 trigeminal neuralgia. J Neurosurg 113: 511-5

Zakrzewska J M (2001): Consumer views on management of trigeminal neuralgia. Headache 41: 369-76

Zakrzewska J M (2002): Diagnosis and differential diagnosis of trigeminal neuralgia. Clin J Pain 18: 14-21

Zakrzewska J M (2007): Diagnosis and management of non-dental orofacial pain. Dent Update 34: 134-6, 138-9

Zakrzewska J M (2010 a): Assessment and treatment of trigeminal neuralgia. Br J Hosp Med (Lond) 71: 490-4

Zakrzewska J M (2010 b): Medical management of trigeminal neuropathic pains. Expert Opin Pharmacother 11: 1239-54

Zakrzewska J M, Patsalos P N (2002): Long-term cohort study comparing medical (oxcarbazepine) and surgical management of intractable trigeminal neuralgia. Pain 95: 259-66

Zakrzewska J M, Coakham H B (2012): Microvascular decompression for trigeminal neuralgia: update. Curr Opin Neurol 25: 296-301 


\section{Danksagung}

Ganz besonders danke ich

meinem Doktorvater, Herrn Prof. Dr. Veit Rohde, für die Überlassung des Themas, meinem Betreuer, Herrn Dr. Kajetan von Eckardstein, für die über Jahre hervorragende Betreuung, Ansprechbarkeit und Hilfbereitschaft und

allen Patienten und Zahnärzten, die mit ihrer Teilnahme diese Studie möglich gemacht haben. 


\section{Lebenslauf}

Am 3.12.1983 wurde ich, Moritz Keil, als erstes Kind von Herrn Dr. Peter Keil und Frau Heidi Keil, geb. Hünninghausen, in Dortmund geboren. Von 1990 bis 1994 besuchte ich in Dortmund die „Olpketal Grundschule“ und von 1994 bis 2003 das „Max-PlanckGymnasium“. Dort erlangte ich 2003 die Allgemeine Hochschulreife.

Im Jahr 2000 besuchte ich für 5 Monate die „Lake Forest Academy“ in Illinois in den USA.

In der Zeit von 2003 bis 2004 leistete ich für 10 Monate meinen Zivildienst bei der Johanniter-Unfall-Hilfe Dortmund ab und schloss im Rahmen dessen sowohl meine Ausbildungen zum Rettungshelfer als auch zum Rettungssanitäter ab.

Im Jahr 2004 begann ich zum Wintersemester das Studium der Zahnmedizin an der Medizinischen Fakultät der Georg-August-Universität in Göttingen. Im Jahr Frühjahr 2009 nahm ich an einer Famulatur in Temuco, Chile, teil.

Mein Studium konnte ich im Sommer 2010 mit dem Staatsexamen erfolgreich abschließen.

2010 begann ich meine Assistenzzeit als Zahnarzt bei meinem Vater in Dortmund, führte sie im Jahr 2011 in der Gemeinschaftspraxis Richter und Dr. Grulich in Eutin fort, wo ich sie im September 2012 abschloss.

Am 10.11.2012 wurde meine Tochter Josefina Keil Villar in Kiel geboren.

Von Dezember 2012 bis Oktober 2013 arbeitete ich als angestellter Zahnarzt in der Praxis Laser und Kollegen in Kiel, bevor mich mein Weg zurück in meine Heimat Dortmund führte. Dort war ich in der Praxis meines Vaters zunächst als Assistent von Oktober bis Dezember 2013 angestellt.

Seit Januar 2014 praktiziere ich als selbstständiger Zahnarzt in der Gemeinschaftspraxis Dr. Peter Keil und Moritz Keil in Dortmund. 